TRANSACTIONS OF THE

AMERICAN MATHEMATICAL SOCIETY

Volume 348, Number 5, May 1996

\title{
DIAGRAMS UP TO COHOMOLOGY
}

\author{
W. G. DWYER AND C. W. WILKERSON
}

\begin{abstract}
We compute (under suitable assumptions) how many ways there are to take a diagram in the homotopy category of spaces and perturb it to get another diagram which looks the same up to cohomology. Sometimes there are no perturbations. This can shed light on the question of whether the $p$ completion of the classifying space of a particular connected compact Lie group is determined up to homotopy by cohomological data.
\end{abstract}

\section{INTRODUCTION}

Let $\mathbf{S p}$ denote the category of spaces, Ho the associated homotopy category, and $\mathbf{D}$ a small (index) category. A diagram in $\mathbf{H o}$ with the shape of $\mathbf{D}$ is by definition a functor $F: \mathbf{D} \rightarrow$ Ho. Given such a diagram one can ask whether or not it has a realization, i.e., a lift to a functor $\mathbf{D} \rightarrow \mathbf{S p}$, and if so, how many realizations there are up to an appropriate kind of equivalence. This question is studied in [4] and [6] for general diagrams and in [7] for the special case of "centric" diagrams (see $\S 6$ ). In this paper we look at a related question. Let $p$ be a fixed prime number and let $\mathrm{H}^{*}$ denote the cohomology functor $\mathrm{H}^{*}\left(-; \mathbf{F}_{p}\right)$. If $F: \mathbf{D} \rightarrow \mathbf{H o}$ is a functor, an $\mathrm{H}^{*}$-clone of $F$ is by definition a collection $\left(G,\left\{s_{d}\right\}\right)$ where

1. $G: \mathbf{D} \rightarrow \mathbf{H o}$ is a functor,

2. for each object $d$ of $\mathbf{D}, s_{d}: F(d) \rightarrow G(d)$ is an isomorphism in Ho, i.e. a homotopy equivalence, and

3. for each morphism $g: d \rightarrow e$ of $\mathbf{D}$, the diagram

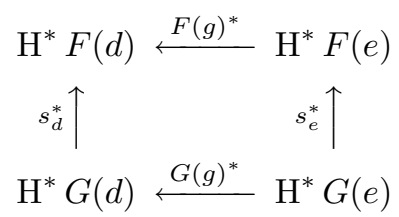

commutes.

In other words, an $\mathrm{H}^{*}$-clone of $F$ is another diagram in the homotopy category which is built from essentially the same spaces as $F$ and looks exactly the same as $F$ from the point of view of mod $p$ cohomology. An equivalence or isomorphism between two $\mathrm{H}^{*}$-clones $\left(G,\left\{s_{d}\right\}\right)$ and $\left(G^{\prime},\left\{s_{d}^{\prime}\right\}\right)$ is a natural equivalence $t: G \rightarrow G^{\prime}$ with the property that for each object $d$ of $\mathbf{D}$, the composite

$$
F(d) \stackrel{s_{d}}{\longrightarrow} G(d) \stackrel{t_{d}}{\longrightarrow} G^{\prime}(d) \stackrel{\left(s_{d}^{\prime}\right)^{-1}}{\longrightarrow} F(d)
$$

Received by the editors September 29, 1994.

1991 Mathematics Subject Classification. Primary 55S99; Secondary 55U99, 55R35, 55R65.

The authors were supported in part by the National Science Foundation 
gives the identity map of $\mathrm{H}^{*} F(d)$. Our main theorem (2.4) shows how to compute the set of isomorphism classes of $\mathrm{H}^{*}$-clones of $F$ in the special case in which $F$ satisfies a "centric" condition (2.1) analogous to that of [7]. We also work out some examples $(\S 3, \S 5)$ and explain (6.7) how our results relate to those of [7].

The motivation for this comes from the following example. Suppose that $\mathcal{X}$ is a $p$-compact group [11], for instance, $\mathcal{X}$ might be the $p$-completion $G_{p}$ of a compact Lie group $G$ such that $\pi_{0} G$ is a finite $p$-group. Given $\mathcal{X}$, the decomposition method of [12] (see also [9] and [13]) produces

1. a category $\mathbf{A}_{\mathcal{X}}$

2. a functor $\alpha_{\mathcal{X}}: \mathbf{A}_{\mathcal{X}}^{\mathrm{op}} \rightarrow \mathbf{S p}$, and

3. a natural homotopy equivalence $\mathrm{B} \mathcal{X} \sim($ hocolim $\alpha \mathcal{X}) \hat{p}$.

Let $\bar{\alpha}_{\mathcal{X}}$ denote the diagram in the homotopy category which underlies $\alpha \mathcal{X}$. We make the following conjecture (see the discussion below).

1.1 Conjecture. Let $\mathcal{X}$ be a p-compact group (perhaps connected). Then $\bar{\alpha}_{\mathcal{X}}$ has no nontrivial $\mathrm{H}^{*}$-clones, i.e., any $\mathrm{H}^{*}$-clone of $\bar{\alpha}_{\mathcal{X}}$ is isomorphic to $\bar{\alpha} \mathcal{X}$.

We show in $\S 3$ that the machinery of this paper applies to $\bar{\alpha}_{X}$; this gives a way to check 1.1 in particular cases.

1.2 Background of Conjecture 1.1. Given a $p$-compact group $\mathcal{X}$, it is interesting to ask whether or not the weak homotopy type of $\mathrm{B} \mathcal{X}$ is determined by $\mathrm{H}^{*} \mathrm{~B} \mathcal{X}$ as an algebra over the mod $p$ Steenrod algebra. The answer to this question is known to be "yes" in many cases [8] [17] although sometimes the answer is "no" (for example, $\mathrm{H}^{*} \mathbf{B Z} / p^{2}$ is isomorphic to $\mathrm{H}^{*} \mathbf{B Z} / p^{3}$ although $\mathbf{B Z} / p^{2}$ and $\mathbf{B Z} / p^{3}$ are not weakly equivalent). Given a specific $\mathcal{X}$, it is tempting to attack this question with an inductive argument that exploits the above decomposition method. Suppose that $\mathcal{Y}$ is a $p$-compact group with $\mathrm{H}^{*} \mathrm{~B} \mathcal{Y}$ isomorphic to $\mathrm{H}^{*} \mathrm{~B} \mathcal{X}$ as an algebra over the Steenrod algebra. To show that $\mathrm{B} \mathcal{Y}$ is weakly equivalent to $\mathrm{B} \mathcal{X}$ it is enough to show that the category $\mathbf{A}_{\mathcal{X}}$ is equivalent to $\mathbf{A}_{\mathcal{Y}}$ in such a way that, under this equivalence, the functors $\alpha_{\mathcal{X}}$ and $\alpha \mathcal{Y}$ are weakly equivalent, i.e., related by a zigzag of natural transformations whose constituent maps are weak equivalences. (Recall that the homotopy colimit construction converts such a natural transformation into a weak equivalence [1, p. 335]). This is not too far-fetched, since choice of an isomorphism $f: \mathrm{H}^{*} \mathrm{~B} \mathcal{X} \cong \mathrm{H}^{*} \mathrm{~B} \mathcal{Y}$ provides both an isomorphism of categories $\mathbf{A}_{f}: \mathbf{A}_{\mathcal{X}} \rightarrow \mathbf{A}_{\mathcal{Y}}$ and a natural equivalence $\mathrm{H}^{*} \alpha_{\mathcal{X}} \cong \mathrm{H}^{*}\left(\alpha_{\mathcal{Y}} \cdot \mathbf{A}_{f}\right)$. The functors $\alpha_{\mathcal{X}}$ and $\alpha_{\mathcal{Y}}$ take on values which are themselves the classifying spaces of $p$-compact groups, and so it is conceivable that there might be inductive information available showing that for each object $v$ of $\mathbf{A}_{X}$ the spaces $\alpha_{\mathcal{X}}(v)$ and $\alpha \mathcal{Y}\left(\mathbf{A}_{f} v\right)$ are determined by their cohomology rings. One would hope to conclude from such inductive information that $\bar{\alpha} \mathcal{Y} \cdot \mathbf{A}_{f}$ is an $\mathrm{H}^{*}$-clone of $\bar{\alpha}_{\mathcal{X}}$, although the reader who tries to work this out will find that there is a technical issue which can be resolved by knowing that certain automorphisms of the rings $\mathrm{H}^{*} \alpha_{\mathcal{X}}(v)$ are induced by self-equivalences of the spaces $\left.\alpha_{\mathcal{X}}(v)\right)$. Given Conjecture 1.1, we would conclude that $\bar{\alpha} \mathcal{Y} \cdot \mathbf{A}_{f}$ and $\bar{\alpha}_{\mathcal{X}}$ are isomorphic. This in itself does not imply the desired result that $\alpha_{\mathcal{Y}} \cdot \mathbf{A}_{f}$ and $\alpha_{\mathcal{X}}$ are weakly equivalent; the problem is that (up to an appropriate equivalence) $\bar{\alpha}_{\mathcal{X}}$ might have more than one lift to a functor $\mathbf{A}_{\mathcal{X}} \rightarrow \mathbf{S p}$. However, the number of such lifts can be computed with the machinery of [7], and we conjecture that for any (connected?) $p$-compact group $\mathcal{X}$ there is only one. 
This proposed line of reasoning factors the analysis of $\alpha_{\mathcal{X}}$ into two parts: studying the number of $\mathrm{H}^{*}$-clones of $\bar{\alpha}_{\mathcal{X}}$ and then the number of realizations of $\bar{\alpha}_{\mathcal{X}}$ by a diagram of spaces. In each case the number that comes up is conjecturally equal to 1 . One could alternatively enumerate directly the "realizations of $\mathrm{H}^{*}$-clones of $\bar{\alpha}_{\mathcal{X}}$ by diagrams of spaces" and hope that there is only one of those. This is the content of Conjecture 6.8. See the remarks after Conjecture 6.8 for some further discussion.

Organization of the paper. Section 2.4 enumerates the set of $\mathrm{H}^{*}$-clones of diagrams of a certain special type, and $\S 3$ shows that this enumeration applies to the case of the decomposition diagram of a $p$-compact group. In $\S 4$ there is a detailed computational analysis of the "lim" " which comes up in the examples from $\S 3$; we use this in $\S 5$ to prove Conjecture 1.1 for the $\mathbf{F}_{2}$-completion of the exceptional Lie group $G_{2}$. Calculations similar to the ones in $\S 5$ also appear in the work of K. Premadasa [19]. Finally, $\S 6$ describes a homotopy limit which combines the ideas here with those of [7] to give, for a suitable diagram in the "cohomology category" an efficient computation of the set of equivalence classes of its realizations. We close with Conjecture 6.8, which, as suggested above, is in a sense a generalization of 1.1 .

Notation and terminology. The category $\mathbf{S p}$ can be taken to be either the category of topological spaces or of simplicial sets. When forming a function space $\operatorname{Map}(A, B)$, we assume in the first case that $A$ has been replaced if necessary by a weakly equivalent cofibrant object (i.e. CW-complex) and in the second that $B$ has been replaced if necessary by a weakly equivalent fibrant object (i.e. Kan complex). The homotopy category $\mathbf{H o}$ is the category obtained from $\mathbf{S p}$ by formally inverting all weak equivalences.

If $A$ is a space, $A_{\hat{p}}$ denotes the $\mathbf{F}_{p}$-completion of $A$ in the sense of [1].

The authors are grateful to the referee for his or her detailed remarks.

\section{THE MAIN THEOREM}

If $f: A \rightarrow B$ is a map in $\mathbf{H o}$, let $[A, B]_{\{f\}}$ denote the set of maps $f^{\prime}: A \rightarrow B$ in Ho such that $\mathrm{H}^{*}\left(f^{\prime}\right)=\mathrm{H}^{*}(f)$.

2.1 Definition. A map $f: A \rightarrow B$ in Ho is said to be $\mathrm{H}^{*}$-centric up to homotopy if

1. $[A, A]_{\{\mathrm{id}\}}$ is a group under composition, and

2. composition with $f$ induces a bijection $[A, A]_{\{\text {id }\}} \cong[A, B]_{\{f\}}$.

A functor $F: \mathbf{D} \rightarrow \mathbf{H o}$ is said to be $\mathrm{H}^{*}$-centric up to homotopy if $F(g)$ satisfies the above conditions for each morphism $g$ of $\mathbf{D}$.

2.2 Remarks. We will define the stronger notion of $\mathrm{H}^{*}$-centric later on (6.3). Note that if $A$ is a space which is $\mathbf{F}_{p}$-complete [1] then $A$ satisfies condition condition 1 above; this follows from the fact that a map between $\mathbf{F}_{p}$-complete spaces is a weak equivalence if and only if it induces an isomorphism on mod $p$ cohomology.

Example. If $\alpha: G_{1} \rightarrow G_{2}$ is a map of finite groups, denote by $\alpha^{*}$ the induced cohomology map $\mathrm{H}^{*}(\mathrm{~B} \alpha)$. Let $i: G \rightarrow H$ be an inclusion of finite $p$-groups, $N(G)$ the normalizer of $G$ in $H$, and $N^{\text {in }}(G) \subset N(G)$ the subgroup of $N(G)$ consisting of elements whose conjugation action on $G$ is via inner automorphisms. Since inner automorphisms of a group act trivially on the cohomology of the classifying space, 
there is a natural homomorphism $N(G) / N^{\text {in }}(G) \rightarrow \operatorname{Aut}\left(\mathrm{H}^{*} \mathrm{~B} G\right)$. It is not hard to see that the map $\mathrm{B} i: \mathrm{B} G \rightarrow \mathrm{BH}$ is $\mathrm{H}^{*}$-centric up to homotopy if and only if

1. for any homomorphism $j: G \rightarrow H$ with $\mathrm{H}^{*}(\mathrm{~B} j)=\mathrm{H}^{*}(\mathrm{~B} i)$ the subgroup $j(G)$ of $H$ is conjugate to $G$, and

2. the map $N(G) / N^{\text {in }}(G) \rightarrow \operatorname{Aut}\left(\mathrm{H}^{*} \mathrm{~B} G\right)$ is injective, and its image contains $\alpha^{*}$ for every automorphism $\alpha$ of $G$ such that $\alpha^{*} i^{*}=i^{*}$.

It follows from 3.1 that these conditions are satisfied if $G$ is the centralizer in $H$ of an elementary abelian $p$-group.

Suppose that $F: \mathbf{D} \rightarrow \mathbf{H o}$ is a functor which is $\mathrm{H}^{*}$-centric up to homotopy. Let Grp denote the category of groups, and construct a functor $\phi_{F}: \mathbf{D}^{\mathrm{op}} \rightarrow \mathbf{G r p}$ by setting

$$
\phi_{F}(d)=[F(d), F(d)]_{\{\mathrm{id}\}}
$$

for each object $d$ of $\mathbf{D}$. For a map $g: d \rightarrow e$ of $\mathbf{D}$ the induced homomorphism $\phi_{F}(g): \phi_{F}(e) \rightarrow \phi_{F}(d)$ assigns to a map $v \in[F(e), F(e)]_{\{\mathrm{id}\}}$ the unique element $u$ of $\phi_{F}(d)$ such that the following diagram

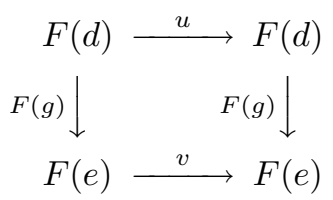

commutes. The existence and uniqueness of $u$ follow immediately from the fact that $F(g)$ is $\mathrm{H}^{*}$-centric up to homotopy. Given elements $v$ and $v^{\prime}$ in $\phi_{F}(e)$, let $u$ and $u^{\prime}$ denote their respective images in $\phi_{F}(d)$ and consider the following diagram

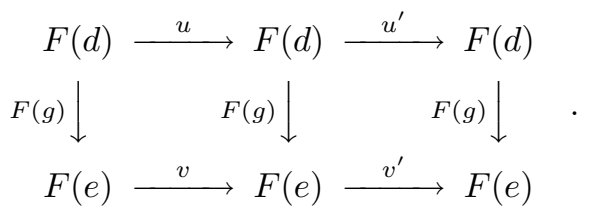

By choice of $u$ and $u^{\prime}$ each small square commutes. It follows that the large square commutes, which shows that the image under $\phi_{F}(g)$ of $v^{\prime} v$ is $u^{\prime} u$ and thus that $\phi_{F}(g)$ is a group homomorphism.

Given a functor $F: \mathbf{D} \rightarrow \mathbf{H o}$, let $\mathcal{C} \ell(F)$ denote the set of equivalence classes ( $\S 1$ ) of $\mathrm{H}^{*}$-clones of $F$. This is a pointed set with the class of $\left(F,\left\{\operatorname{id}_{d}\right\}\right)$ as basepoint.

2.4 Theorem. If $F: \mathbf{D} \rightarrow \mathbf{H o}$ is a functor which is $\mathrm{H}^{*}$-centric up to homotopy, then there is a natural bijection of pointed sets

$$
\mathcal{C} \ell(F) \cong \lim ^{1} \phi_{F}
$$

and a natural isomorphism of groups

$$
\operatorname{Aut}\left(F,\left\{\operatorname{id}_{d}\right\}\right) \cong \lim \phi_{F} .
$$

Remark. In the above statement, $\operatorname{Aut}\left(F,\left\{\operatorname{id}_{d}\right\}\right)$ stands for the group of self-equivalences or self-isomorphisms of the (trivial) $\mathrm{H}^{*}$-clone $\left(F,\left\{\mathrm{id}_{d}\right\}\right)$ of $F$ (see $\S 1$ ). 
2.5 Description of $\lim ^{1}$. Before proving 2.4 we will give an explicit description of the functor "lim" (also see [1, p. 307]). Suppose that D is a small category and that $\phi: \mathbf{D}^{\text {op }} \rightarrow \mathbf{G r p}$ is a functor. For convenience, if $g: d_{0} \rightarrow d_{1}$ is a morphism of $\mathbf{D}$ we will write $g^{\sharp}: \phi\left(d_{1}\right) \rightarrow \phi\left(d_{0}\right)$ for the map $\phi(g)$. Let $\mathcal{O}$ denote the set of objects of $\mathbf{D}, \mathcal{M}$ the set of morphisms of $\mathbf{D}$, and $\mathcal{S}$ the disjoint union

$$
\mathcal{S}=\bigcup_{d \in \mathcal{O}} \phi(d)
$$

The object $\mathcal{S}$ is just a set, or, perhaps better, the collection of morphisms in a groupoid with object set $\mathcal{O}$. Let $C^{0}(\phi)$ denote the set of all functions $c: \mathcal{O} \rightarrow \mathcal{S}$ such that for each $d \in \mathcal{O}, c(d) \in \phi(d)$. It is clear that $C^{0}(\phi)$ is a group under pointwise multiplication. Let $C^{1}(\phi)$ denote the set of all functions $c: \mathcal{M} \rightarrow \mathcal{S}$ such that for each $g: d_{0} \rightarrow d_{1}$ in $\mathcal{M}, c(g) \in \phi\left(d_{0}\right)$. An element $c \in C^{1}(\phi)$ is called a "normalized 1-cocycle" if

1. for each identity map $g: d_{0} \rightarrow d_{0}, c(g)$ is the identity element of $\phi\left(d_{0}\right)$, and

2. for each pair $g_{0}: d_{0} \rightarrow d_{1}$ and $g_{1}: d_{1} \rightarrow d_{2}$ of composable elements of $\mathcal{M}$, the product

$$
g_{0}^{\sharp}\left(c\left(g_{1}\right)\right) c\left(g_{1} g_{0}\right)^{-1} c\left(g_{0}\right)
$$

is the identity element of $\phi\left(d_{0}\right)$.

Let $Z^{1}(\phi) \subset C^{1}(\phi)$ denote the collection of normalized 1-cocycles; this is a pointed set with basepoint given by the element $z \in Z^{1}(\phi)$ which assigns to each $g: d_{0} \rightarrow d_{1}$ in $\mathcal{M}$ the identity element of $\phi\left(d_{0}\right)$. There is an action of $C^{0}(\phi)$ on $Z^{1}(\phi)$ such that if $c \in C^{0}(\phi)$ and $z \in Z^{1}(\phi)$ then $c \cdot z=z^{\prime}$, where for each $g: d_{0} \rightarrow d_{1}$ in $\mathcal{M}$,

$$
z^{\prime}(g)=c\left(d_{0}\right) z(g) g^{\sharp}\left(c\left(d_{1}\right)^{-1}\right) .
$$

By definition, the orbit set of this action is $\lim ^{1} \phi$. The basepoint of $Z^{1}(\phi)$ projects to a basepoint for $\lim ^{1} \phi$.

Proof of 2.4. Say that an $\mathrm{H}^{*}$-clone $\left(G,\left\{s_{d}\right\}\right)$ of $F$ is special if for each object $d$ of $\mathbf{D}, G(d)=F(d)$ and $s_{d}$ is the identity map of $F(d)$. It is immediate that any $\mathrm{H}^{*}$-clone $\left(G^{\prime},\left\{s_{d}^{\prime}\right\}\right)$ of $F$ is equivalent to a special $\mathrm{H}^{*}$-clone $\left(G,\left\{s_{d}\right\}\right)$; for instance, set $G(g)=\left(s_{d_{1}}^{\prime}\right)^{-1} G^{\prime}(g) s_{d_{0}}^{\prime}$ for each morphism $g: d_{0} \rightarrow d_{1}$ of $\mathbf{D}$. It follows that we can interpret $\mathcal{C} \ell(F)$ as the set of equivalence classes of special $\mathrm{H}^{*}$-clones of $F$. If $G$ is a special $\mathrm{H}^{*}$-clone of $F$, let $z_{G} \in C^{1}\left(\phi_{F}\right)$ be the function which assigns to each morphism $g: d_{0} \rightarrow d_{1}$ of $\mathbf{D}$ the unique element $h \in\left[F\left(d_{0}\right), F\left(d_{0}\right)\right]_{\{\text {id }\}}$ which makes the following diagram commute

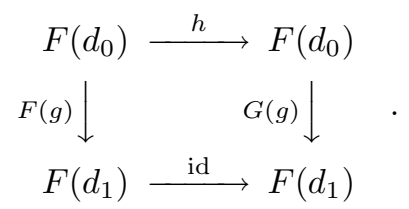

The existence and uniqueness of $h$ follows immediately from the fact that $F(g)$ is $\mathrm{H}^{*}$-centric up to homotopy. Clearly $z_{G}(g)$ is the appropriate identity element if $g$ 
is an identity map. The commutative diagram

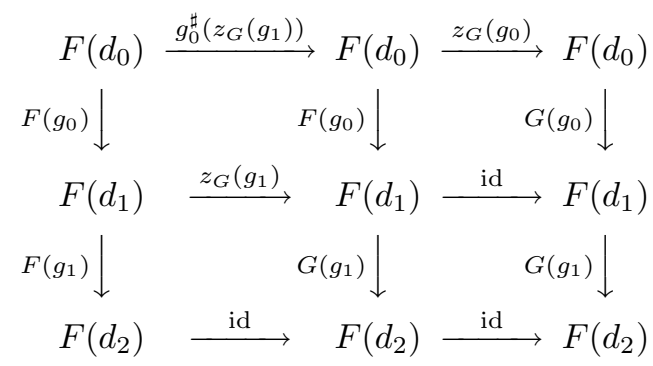

shows that $z_{G}\left(g_{1} g_{0}\right)=z_{G}\left(g_{0}\right) g_{0}^{\sharp}\left(z_{G}\left(g_{1}\right)\right)$ and thus that $z_{G} \in Z^{1}\left(\phi_{F}\right)$. It is easy to check directly that the image $\left\langle z_{G}\right\rangle$ of $z_{G}$ in $\lim ^{1} \phi_{F}$ depends only on the equivalence class of the special $\mathrm{H}^{*}$-clone $G$, and that the assignment $G \mapsto\left\langle z_{G}\right\rangle$ gives the desired bijection $\mathcal{C} \ell(F) \rightarrow \lim ^{1} \phi_{F}$. The identification of $\lim \phi_{F}$ is straightforward.

\section{The Basic EXAmple}

In this section we will give the motivating example of a diagram which is $\mathrm{H}^{*}$ centric up to homotopy. Suppose that $\mathcal{X}$ is a $p$-compact group with classifying space $\mathrm{B} \mathcal{X}$. Let $\mathbf{A}_{\mathcal{X}}$ denote the category whose objects are the pairs $(V, \mathrm{~B} f)$, where $V$ is a nontrivial elementary abelian $p$-group and $f: V \rightarrow \mathcal{X}$ is a conjugacy class of monomorphisms $[12, \S 8]$. By definition, giving $f$ amounts to giving an ordinary homotopy class of maps $\mathrm{B} f: \mathrm{B} V \rightarrow \mathrm{B} \mathcal{X}$ such that $\mathrm{H}^{*} \mathrm{~B} V$ is finitely generated as a module over $(\mathrm{B} f)^{*}\left(\mathrm{H}^{*} \mathrm{~B} \mathcal{X}\right)$. A morphism $(V, f) \rightarrow\left(V^{\prime}, f^{\prime}\right)$ in $\mathbf{A}_{\mathcal{X}}$ is an injection $i: V \rightarrow V^{\prime}$ such that $f^{\prime} \cdot i$ is conjugate to $f$ (equivalently, $\left(\mathrm{B} f^{\prime}\right) \cdot(\mathrm{B} i)$ is homotopic to $\mathrm{B} f$ ). There is a functor $\alpha_{\mathcal{X}}: \mathbf{A}_{\mathcal{X}}^{\mathrm{op}} \rightarrow \mathbf{S p}$ given by

$$
\alpha_{\mathcal{X}}(V, f)=\operatorname{Map}(\mathrm{B} V, \mathrm{~B} \mathcal{X})_{\mathrm{B} f}
$$

where the subscript "B $f$ " denotes the mapping space component corresponding to the homotopy class $\mathrm{B} f$. This diagram is interesting because there is a natural map hocolim $\alpha_{\mathcal{X}} \rightarrow \mathrm{B} \mathcal{X}$ which induces an isomorphism on $\bmod p$ homology as well as a weak equivalence $\left(\right.$ hocolim $\left.\alpha_{\mathcal{X}}\right) \hat{p} \sim \mathrm{B} \mathcal{X}[12,8.1]$.

Let $\bar{\alpha}_{\mathcal{X}}: \mathbf{A}_{\mathcal{X}}^{\text {op }} \rightarrow$ Ho denote the diagram in the homotopy category which underlies $\alpha_{\mathcal{X}}$.

3.1 Theorem. For any p-compact group $\mathcal{X}$ the diagram $\bar{\alpha}_{\mathcal{X}}$ is $\mathrm{H}^{*}$-centric up to homotopy.

The proof depends on three lemmas. In these lemmas, $Z$ denotes a $p$-compact group, $V$ an elementary abelian $p$-group, and $i: V \rightarrow Z$ a monomorphism (see above). Let $\mathrm{B} Y$ denote the mapping space component $\operatorname{Map}(\mathrm{B} V, \mathrm{~B} Z)_{\mathrm{B} i}$. (The space $Y=\Omega \mathrm{B} Y$ is a $p$-compact group which is called the centralizer of $V$ in $Z$.) Evaluation at the basepoint of $\mathrm{B} V$ gives a map $\mathrm{B} j: \mathrm{B} Y \rightarrow \mathrm{B} Z$. The map $\mathrm{B} i$ lifts to a map $\mathrm{B} i^{\prime}: \mathrm{B} V \rightarrow \mathrm{B} Y$ such that $(\mathrm{B} j) \cdot\left(\mathrm{B} i^{\prime}\right)=\mathrm{B} i$. This lift has two key properties.

- The map $\mathrm{B} i^{\prime}$ is central $[12,2.7]$ in the sense that evaluation at the basepoint of $\mathrm{B} V$ gives a weak equivalence

$$
\operatorname{Map}(\mathrm{B} V, \mathrm{~B} Y)_{\mathrm{B} i^{\prime}} \stackrel{\sim}{\longrightarrow} \mathrm{B} Y .
$$


- The map $\mathrm{B} i^{\prime}$ extends to a principal fibration sequence $[12,2.8]$

$$
\mathrm{B} V \stackrel{\mathrm{B} i^{\prime}}{\longrightarrow} \mathrm{B} Y \rightarrow \mathrm{B}(Y / V) .
$$

The lift $\mathrm{B} i^{\prime}$ is obtained from the usual abelian group structure on $\mathrm{B} V[11,8.2]$, and sends $y \in \mathrm{B} V$ to the map $\mathrm{B} V \rightarrow \mathrm{B} \mathcal{X}$ which takes $x \in \mathrm{B} V$ to $\mathrm{B} j(x+y)$.

3.4 Lemma. Consider the commutative diagram

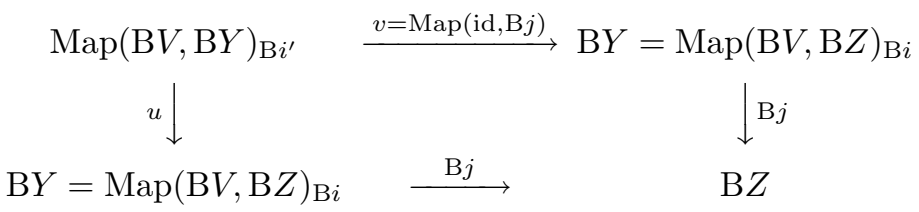

in which the vertical maps are given by evaluation at the basepoint of $\mathrm{B} V$. Then the maps $u$ and $v$ are homotopy equivalences and, if $u^{-1}$ is the inverse of $u$ in $\mathbf{H o \text { , }}$ the composite $v u^{-1}$ is equal in $\mathbf{H o}$ to the identity map of $Y$.

Proof. Let $k$ be the composite of $\mathrm{B} i$ with the map $\mu: \mathrm{B} V \times \mathrm{B} V=\mathrm{B}(V \times V) \rightarrow \mathrm{B} V$ induced by addition $V \times V \rightarrow V$. The space in the upper left hand corner of 3.5 can be identified as $\operatorname{Map}(\mathrm{B} V \times \mathrm{B} V, \mathrm{~B} Z)_{k}$. Under this identification the map $u$ corresponds to restriction to the factor $* \times \mathrm{B} V$ and $v$ corresponds to restriction to the factor $\mathrm{B} V \times *$. Composition with $\mu$ thus gives a map

$$
w: \operatorname{Map}(\mathrm{B} V, \mathrm{~B} Z)_{\mathrm{B} i} \rightarrow \operatorname{Map}(\mathrm{B} V \times \mathrm{B} V, \mathrm{~B} Z)_{k}
$$

such that $u w$ and $v w$ are identity maps. The map $u$ is an equivalence as above (3.2); this implies that $w$ and hence $v$ are also equivalences. The composite $v \cdot u^{-1}$ is then the same in Ho as the composite $(v w)(u w)^{-1}$ of two identity maps.

Let $\operatorname{Map}(\mathrm{B} Y, \mathrm{~B} Y)_{\left[\mathrm{B} i^{\prime}\right]}$ denote the space of all maps $h: \mathrm{B} Y \rightarrow \mathrm{B} Y$ such that $h \cdot\left(\mathrm{B} i^{\prime}\right)$ is homotopic to $\mathrm{B} i^{\prime}$. Similarly, let $\operatorname{Map}(\mathrm{B} Y, \mathrm{~B} Z)_{[\mathrm{B} i]}$ denote the space of all maps $h: \mathrm{B} Y \rightarrow \mathrm{B} Z$ such that $h \cdot(\mathrm{B} i)$ is homotopic to $\mathrm{B} i^{\prime}$.

3.6 Lemma. The map $\mathrm{B} j: \mathrm{B} Y \rightarrow \mathrm{B} Z$ induces an equivalence

$$
\operatorname{Map}(\mathrm{B} Y, \mathrm{~B} Y)_{\left[\mathrm{B} i^{\prime}\right]} \stackrel{\sim}{\longrightarrow} \operatorname{Map}(\mathrm{B} Y, \mathrm{~B} Z)_{[\mathrm{B} i]} .
$$

Proof. Since $(\mathrm{B} j) \cdot\left(\mathrm{B} i^{\prime}\right)=\mathrm{B} i$, it is clear that composition with $\mathrm{B} j$ gives a map of the indicated type. Recall that if $f: E \rightarrow B$ is a fibration over a connected base $B$ with fibre $F$, and $X$ is some target space, then there is a derived fibration $f_{X}: E_{X} \rightarrow B$ with fibre $\operatorname{Map}(F, X)$ such that the space of sections of $f_{X}$ is equivalent to $\operatorname{Map}(E, X)$. (There is a discussion of this construction in [2, §3].) Applying this observation to the fibration 3.3 and restricting to suitable components gives a fibration $p_{1}: E_{1} \rightarrow \mathrm{B}(Y / V)$ with fibre $\operatorname{Map}(\mathrm{B} V, \mathrm{~B} Y)_{\mathrm{B} i^{\prime}}$ and space of sections $\operatorname{Map}(\mathrm{B} Y, \mathrm{~B} Y)_{\left[\mathrm{B} i^{\prime}\right]}$. There is a similar fibration $p_{2}: E_{2} \rightarrow \mathrm{B}(Y / V)$ with fibre $\operatorname{Map}(\mathrm{B} V, \mathrm{~B} Z)_{\mathrm{B} i}$ and space of sections $\mathrm{Map}(\mathrm{B} Y, \mathrm{~B} Z)_{[\mathrm{B} i]}$. The map $\mathrm{B} j$ induces a map between these two fibrations which by 3.4 is a fibrewise equivalence; it follows that the corresponding map on spaces of sections is an equivalence. 
3.7 Lemma. The map $\mathrm{B} j: \mathrm{B} Y \rightarrow \mathrm{B} Z$ is $\mathrm{H}^{*}$-centric up to homotopy.

Proof. Since homotopy classes of maps from $\mathrm{B} V$ to $\mathrm{B} Z$ or $\mathrm{B} Y$ are detected by their effect on mod $p$ cohomology [15, 3.1.4], it is clear that there is a commutative diagram

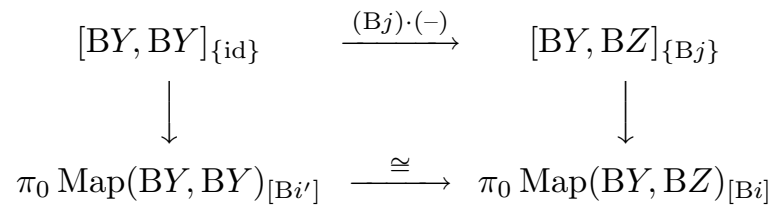

in which the vertical arrows are monomorphisms. By 3.6, the lower horizontal arrow is a bijection. To show that $\mathrm{B} j$ is $\mathrm{H}^{*}$-centric up to homotopy it is enough to show that the upper horizontal arrow is a surjection.

Let $h: \mathrm{B} Y \rightarrow \mathrm{B} Z$ be a map which has the same effect on mod $p$ cohomology as $\mathrm{B} j$; it is necessary to find a map $w: \mathrm{B} Y \rightarrow \mathrm{B} Y$ which induces the identity map on $\bmod p$ cohomology and such that $(\mathrm{B} j) \cdot w$ is homotopic to $h$. Consider the commutative diagram

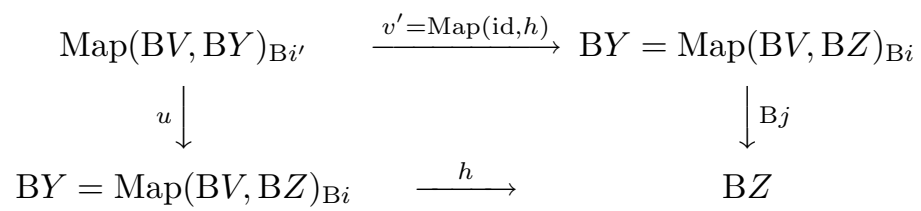

in which the vertical arrows are given by evaluation at the basepoint of $\mathrm{B} V$. The map $u$ is an equivalence by 3.4. We will be done if we can show both that the map $v^{\prime}$ gives an isomorphism on mod $p$ cohomology and that the composite $v^{\prime} u^{-1}$ induces the identity map on $\mathrm{H}^{*} \mathrm{~B} Y$; the composite $w=v^{\prime} u^{-1}$ will then be the required map with $(\mathrm{B} j) \cdot w$ homotopic to $h$. Let $\mathcal{K}$ denote the category of unstable algebras over the $\bmod p$ Steenrod algebra and $T(V,-): \mathcal{K} \rightarrow \mathcal{K}$ the functor which is left adjoint to tensor product with $\mathrm{H}^{*} \mathrm{~B} V$. For a map $\gamma: \mathrm{H}^{*} \mathrm{~B} V \rightarrow R$ in $\mathcal{K}$, let $T(V, R)_{\gamma}$ denote the summand or "component" of $T(V, R)$ corresponding to $\gamma[9$, $\S 3]$. The inclusion $0 \rightarrow V$ induces a natural map $e_{\gamma}: T(0, R)=R \rightarrow T(V, R)_{\gamma}$. If $A$ is a space, $g: \mathrm{B} V \rightarrow A$ is a map, and $\gamma=g^{*}: \mathrm{H}^{*} A \rightarrow \mathrm{H}^{*} \mathrm{~B} V$, there is a natural commutative diagram

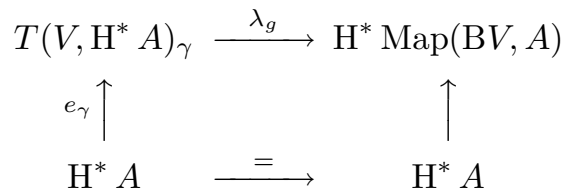

in which the right hand vertical arrow is induced by evaluation at the basepoint of $\mathrm{B} V$. The map $\lambda_{g}$ is an isomorphism if, for instance, $A$ is the classifying space of a $p$-compact group [12, proof of 8.1]. It is a consequence of this fact and of the naturality of 3.9 that the diagram obtained by applying the functor $\mathrm{H}^{*}(-)$ to 3.8 is identical to the diagram obtained by applying $\mathrm{H}^{*}(-)$ to 3.5 (note that there are no choices of isomorphisms here; the diagrams are the same). The desired properties of $v^{\prime}$ follow from 3.4.

Proof of 3.1. The values of the functor $\bar{\alpha}_{\mathcal{X}}$ are $\mathbf{F}_{p}$-complete spaces because they are the classifying spaces of $p$-compact groups $[12,2.5]$. Let $\iota:(V, f) \rightarrow\left(V^{\prime}, f^{\prime}\right)$ be 
a morphism in $\mathbf{A}_{\mathcal{X}}$ coming from an injection $i: V \rightarrow V^{\prime}$. Write $V^{\prime}=V^{\prime \prime} \times i(V)$, and let $\mathrm{B} f^{\prime \prime}: \mathrm{B} V^{\prime \prime} \rightarrow \operatorname{Map}(\mathrm{B} V, \mathrm{~B} \mathcal{X})_{\mathrm{B} f}$ be the map which is adjoint to $\mathrm{B} f^{\prime}$ : $\mathrm{B} V^{\prime \prime} \times \mathrm{B} V \rightarrow \mathrm{B} \mathcal{X}$. The map $\alpha \mathcal{X}(\iota)$ can be identified as the map

$$
\operatorname{Map}\left(\mathrm{B} V^{\prime}, \mathrm{B} \mathcal{X}\right)_{\mathrm{B} f^{\prime}}=\operatorname{Map}\left(\mathrm{B} V^{\prime \prime}, \operatorname{Map}(\mathrm{B} V, \mathrm{~B} \mathcal{X})_{\mathrm{B} f}\right)_{\mathrm{B} f^{\prime \prime}} \rightarrow \operatorname{Map}(\mathrm{B} V, \mathrm{~B} \mathcal{X})_{\mathrm{B} f}
$$

obtained by evaluating at the basepoint of $\mathrm{B} V^{\prime \prime}$. Since $\operatorname{Map}(\mathrm{B} V, \mathrm{~B} \mathcal{X})$ is the classifying space of a $p$-compact group $[11,5.1]$, it follows from 3.7 that $\alpha_{\mathcal{X}}(\iota)$ is $\mathrm{H}^{*}$-centric up to homotopy.

\section{VANISHING $\lim ^{1}$}

In this section we will give a simple way to check for vanishing of the $\lim ^{1}$ sets that arise in applications of 2.4 to the theory of $p$-compact groups. The formulas in this section can be interpreted as nonabelian generalizations of formulas of Oliver [18].

The first step, which is mostly for notational convenience, is to reduce to certain very explicit categories. For each $i \geq 1$ let $A_{i}$ denote the $\mathbf{F}_{p}$ vector space $\left(\mathbf{F}_{p}\right)^{i}$ and for $n \geq 1$ let $\mathbf{A}_{n}$ denote the category whose objects are the vector spaces $A_{i}$, $1 \leq i \leq n$, and whose morphisms are $\mathbf{F}_{p}$-module monomorphisms. Suppose that $\mathcal{X}$ is a $p$-compact group and that $\phi: \mathbf{A}_{\mathcal{X}} \rightarrow \mathbf{G r p}$ is a functor (for instance, $\phi$ might be the functor derived from $\bar{\alpha}_{\mathcal{X}}$ (3.1) by the procedure of $\S 2$ ). Let $n$ be the largest integer such that there is a monomorphism $A_{n} \rightarrow \mathcal{X}$ (see $\S 3$ ); for the existence of such an $n$ see $[12,8.3]$. Let $\Phi: \mathbf{A}_{n} \rightarrow \mathbf{G r p}$ be the functor given by the formula

$$
\Phi(A)=\prod_{f} \phi(A, f),
$$

in which the product is taken over the $\operatorname{set} \operatorname{Mono}(A, \mathcal{X})$ of all monomorphisms $f$ : $A \rightarrow \mathcal{X}$. The behavior of $\Phi$ on a morphism $i: A \rightarrow B$ of $\mathbf{A}_{n}$ is as follows: the (algebraic) monomorphism $i$ induces a map

$$
i^{*}: \operatorname{Mono}(B, \mathcal{X}) \rightarrow \operatorname{Mono}(A, \mathcal{X})
$$

and, given $x=\left\{x_{f}\right\} \in \Phi(A)$, the image of $x$ under $\Phi(i)$ is the element $y=\left\{y_{g}\right\} \in$ $\Phi(B)$ with $y_{g}=\phi(i)\left(x_{i^{*}(g)}\right)$.

4.1 Lemma. In the above situation, there is a natural isomorphism of pointed sets between $\lim ^{1} \Phi$ and $\lim ^{1} \phi$.

Proof (cf. $[9,2.3]$ ). Let $\phi^{\prime}$ be the restriction of $\phi$ to the full subcategory $\mathbf{A}_{\mathcal{X}}^{\prime}$ of $\mathbf{A}_{\mathcal{X}}$ given by the objects $(V, f)$ such that $V$ is an object of $\mathbf{A}_{n}$. It is clear that the inclusion $\mathbf{A}_{\mathcal{X}}^{\prime} \rightarrow \mathbf{A}_{\mathcal{X}}$ is an equivalence of categories, and so $\lim ^{1} \phi^{\prime}$ is naturally isomorphic to $\lim ^{1} \phi$. By inspection, however, the set $Z^{1}\left(\phi^{\prime}\right)$ corresponds bijectively to $Z^{1}(\Phi)$ in a way which respects the equivalence relations giving $\lim ^{1}$.

From now on, then, we will deal only with functors $\Phi: \mathbf{A}_{n} \rightarrow$ Grp. Let $\Phi_{1}$ be the restriction of $\Phi$ to the subcategory $\mathbf{A}_{1}$ of $\mathbf{A}_{n}$. To simplify both the exposition and the formulas we will assume that $n \geq 3$ and that $\lim ^{1} \Phi_{1}=*$. This last condition is always satisfied if $p=2$, since in this case $\mathbf{A}_{1}$ is a trivial category; the condition is also frequently satisfied for $p$ odd, since $\mathbf{A}_{1}$ is then the category of a 
group of order prime to $p$ (i.e. $\mathrm{GL}\left(1, \mathbf{F}_{p}\right)$ ) and in examples arising from 2.4 the group $\Phi\left(A_{1}\right)$ is usually $p$-complete in some sense.

Let $e^{i}: A_{i} \rightarrow A_{i+1}$ be the standard inclusion obtained by adding a zero as the last coordinate and, for $j>i$, let $e^{i, j}: A_{i} \rightarrow A_{j}$ be the composite $e^{j-1} \cdots e^{i}$. For $j>i$ we will identify $A_{i}$ with its image in $A_{j}$ under $e^{i, j}$. Let $P(i, j) \subset \mathrm{GL}\left(i+j, \mathbf{F}_{p}\right)$ be the subgroup of linear transformations which carry the subspace $A_{i}$ to itself; these are block matrices of the form

$$
\left(\begin{array}{cc}
M_{1} & M_{2} \\
0 & M_{3}
\end{array}\right)
$$

in which $M_{1}$ is square of size $i \times i$. There is a surjection $r_{i}: P(i, j) \rightarrow \mathrm{GL}\left(i, \mathbf{F}_{p}\right)$ which maps the above block matrix to $M_{1}$.

Recall that the Tits building $T\left(A_{3}\right)$ of $A_{3}$ is the graph with a vertex for each 1-dimensional subspace $V$ or 2-dimensional subspace $W$ of $A_{3}$ and an edge for each pair $(V, W)$ with $V \subset W$.

4.2 Definition. A 1-cycle in $T\left(A_{3}\right)$ is a circular sequence of vertices of $T\left(A_{3}\right)$ such that any two adjacent ones are connected by an edge; equivalently, $C$ is a a sequence

$$
C=\left(V_{0}, W_{1}, V_{1}, W_{2}, V_{2}, \ldots, W_{k}, V_{k}\right)
$$

of subspaces of $A_{3}$ such that each $V_{i}$ has dimension 1 , each $W_{i}$ has dimension 2, $W_{i} \supset V_{i-1}, W_{i} \supset V_{i}$, and $V_{k}=V_{0}$. A polarization of $C$ is a collection $\left\{\alpha_{i}, \beta_{i}\right\}_{i=1}^{k}$ of elements of $\mathrm{GL}\left(3, \mathbf{F}_{p}\right)$ such that $\alpha_{i}$ carries the subspace pair $\left(W_{i}, V_{i-1}\right)$ to the standard pair $\left(A_{2}, A_{1}\right)$, and $\beta_{i}$ carries $\left(W_{i}, V_{i}\right)$ to $\left(A_{2}, A_{1}\right)$. The difference elements $\left\{a_{i}, b_{i}\right\}(i=1, \cdots, k)$ associated to the polarization are given by $b_{i}=\beta_{i} \alpha_{i}^{-1}$, $a_{i}=\alpha_{i+1} \beta_{i}^{-1}(i<k)$, and $a_{k}=\alpha_{1} \beta_{k}^{-1}$. Note that $a_{i} \in P(1,2)$ and $b_{i} \in P(2,1)$.

Suppose that $n \geq 3$ and that $\Phi: \mathbf{A}_{n} \rightarrow \mathbf{G r p}$ is a functor. If $x \in \Phi\left(A_{i}\right)$ and $f: \Phi_{i} \rightarrow \Phi_{j}$ is a morphism of $\mathbf{A}_{n}$, write $f_{\#}(x)$ for the image of $x$ under $\Phi(f)$. Observe that $\mathrm{GL}\left(i, \mathbf{F}_{p}\right)$ is the group of self-maps in $\mathbf{A}_{n}$ of $A_{i}(i \leq n)$ so that there is an action of $\operatorname{GL}\left(i, \mathbf{F}_{p}\right)$ on $\Phi\left(A_{i}\right)$ which sends $(g, x)$ to $g_{\#}(x)$.

4.4 Definition. The set $Z_{\mathrm{sp}}^{1}(\Phi)$ of special 1-cocycles for $\Phi$ is the collection of set maps $\zeta: \operatorname{GL}\left(2, \mathbf{F}_{p}\right) \rightarrow \Phi\left(A_{2}\right)$ which have the following three properties.

1. For $g, h \in \mathrm{GL}\left(2, \mathbf{F}_{p}\right), \zeta(g h)=\zeta(g) h_{\#}(\zeta(h))$.

2. If $g \in P(1,1)$, then $\zeta(g)=1$.

3. Suppose that $C$ is a 1 -cycle of $T\left(A_{3}\right)$ (4.3) with polarization $\left\{\alpha_{i}, \beta_{i}\right\}$ and associated difference elements $\left\{a_{i}, b_{i}\right\}$. Let $\tilde{\zeta}$ denote the composite function

$$
P(2,1) \stackrel{r_{2}}{\longrightarrow} \mathrm{GL}\left(2, \mathbf{F}_{p}\right) \stackrel{\zeta}{\longrightarrow} \Phi\left(A_{2}\right) \stackrel{e_{\#}^{2}}{\longrightarrow} \Phi\left(A_{3}\right)
$$

and for each $i$ let $\gamma_{i}$ denote the map $\left(\alpha_{1} \beta_{i}^{-1}\right)_{\#}$. Then the following equality holds in $\Phi\left(A_{3}\right)$ :

$$
\gamma_{k}\left(\tilde{\zeta}\left(b_{k}\right)\right) \gamma_{k-1}\left(\tilde{\zeta}\left(b_{k-1}\right)\right) \cdots \gamma_{1}\left(\tilde{\zeta}\left(b_{1}\right)\right)=1 .
$$


Remark. It can be checked that given conditions 1 and 2 of 4.4, condition 3 holds for all polarizations of a 1-cycle $C$ if and only if it holds for any single polarization.

Let $x$ be an element of the fixed set $F$ of the action of $\mathrm{GL}\left(1, \mathbf{F}_{p}\right)$ on $\Phi\left(A_{1}\right)$, and let $y \in \Phi\left(A_{2}\right)$ be the image of $x$ under $e_{\#}^{1}$. Given $\zeta \in Z_{\mathrm{sp}}^{1}(\Phi)$, there is another element $\zeta^{\prime} \in Z_{\mathrm{sp}}^{1}(\Phi)$ with $\zeta^{\prime}(g)=y \zeta(g) g_{\#}(y)^{-1}$ and the formula $x \cdot \zeta=\zeta^{\prime}$ gives an action of $F$ on $Z_{\mathrm{sp}}^{1}(\Phi)$. Let $Z_{\mathrm{sp}}^{1}(\Phi) / \sim$ denote the orbit set of this action; this has a basepoint given by the orbit of the element $\zeta \in Z_{\mathrm{sp}}^{1}(\Phi)$ with $\zeta(g)=1$ for all $g \in \mathrm{GL}\left(2, \mathbf{F}_{p}\right)$.

4.5 Proposition. Suppose that $\Phi: \mathbf{A}_{n} \rightarrow \operatorname{Grp}$ is a functor, $n \geq 2$. Let $\Phi_{1}$ be the restriction of $\Phi$ to $\mathbf{A}_{1}$, and assume that $\lim ^{1} \Phi_{1}=*$. Then there is a natural monomorphism of pointed sets

$$
\lim ^{1} \Phi \rightarrow Z_{\mathrm{sp}}^{1}(\Phi) / \sim
$$

Remark. In fact, the monomorphism in 4.5 is an isomorphism, although we will not write down the proof of this. The argument consists in following what appears below and observing, with the help of the action of $\mathrm{GL}\left(3, \mathbf{F}_{p}\right)$ on $T\left(A_{3}\right)$, that $\mathrm{GL}\left(3, \mathbf{F}_{p}\right)$ is the quotient of an amalgamated sum $P(1,2) *_{P(1,1,1)} P(2,1)$ by a normal subgroup isomorphic to the fundamental group of $T\left(A_{3}\right)$. For $i>3, \mathrm{GL}\left(i, \mathbf{F}_{p}\right)$ is isomorphic to the appropriate analog of this amalgamated sum, essentially because $T\left(A_{i}\right)$ is 1-connected.

The proof of 4.5 relies on the following more elementary fact from linear algebra.

4.6 Lemma. If $n \geq 3$ then the group $\mathrm{GL}\left(n, \mathbf{F}_{p}\right)$ is generated by the subgroups $P(i, j), i+j=n, i, j>0$.

Proof of 4.5. Let $z$ be an element of $Z^{1}(\Phi)$. According to 2.5 (adjusted for the fact that $\Phi$ is a covariant functor) $z$ is a function which assigns to each morphism $g: A_{i} \rightarrow A_{j}$ of $\mathbf{A}_{n}$ an element $z(g) \in \Phi\left(A_{j}\right)$, such that $z(g)=1$ if $g$ is an identity morphism and

$$
z(g h)=z(g) g_{\#} z(h)
$$

If $c \in C^{0}(\Phi)$, then $z$ represents the same element of $\lim ^{1} \Phi$ as the "cohomologous" cocycle $c \cdot z=z^{\prime}$ with

$$
z^{\prime}(g)=c\left(A_{j}\right) z(g) g_{\#} c\left(A_{i}\right)^{-1}
$$

Since $\lim ^{1} \Phi_{1}=*$ the restriction of $z$ to $\mathbf{A}_{1}$ is cohomologous to the trivial cocycle; in other words, there is an element $x \in \Phi\left(A_{1}\right)$ such that $z(g)=x g_{\#}(x)^{-1}$ for each $g \in \mathrm{GL}\left(1, \mathbf{F}_{p}\right)$. Define an element $c \in C^{0}(\Phi)$ inductively by setting $c\left(A_{1}\right)=x^{-1}$ and $c\left(A_{i+1}\right)$ equal to the inverse of $z\left(e^{i}\right) e_{\#}^{i}\left(c\left(A_{i}\right)\right)$. A calculation with 4.8 shows that the cocycle $c \cdot z=z^{\prime}$ satisfies two conditions:

1. $z^{\prime}(g)=1$ for each $g \in \mathrm{GL}\left(1, \mathbf{F}_{p}\right)$, and

2. $z^{\prime}\left(e^{i}\right)=1(i=1, \ldots, n-1)$. 
Let $Z^{\prime} \subset Z^{1}(\Phi)$ denote the collection of all cocycles which satisfy the above two conditions. Let $F$ denote the fixed set of the action of $\operatorname{GL}\left(1, \mathbf{F}_{p}\right)$ on $\Phi\left(A_{1}\right)$, and, given $x \in F$, let $c_{x} \in C^{0}(\Phi)$ be defined inductively by $c\left(A_{1}\right)=x, c\left(A_{i+1}\right)=$ $e_{\#}^{i} c\left(A_{i}\right)$. The construction $(x, z) \mapsto c_{x} \cdot z$ gives an action of $F$ on $Z^{\prime}$, and by elementary calculation the subgroup $\left\{c_{x}: x \in F\right\}$ of $C^{0}(\Phi)$ is equal to the set of elements $c \in C^{0}(\Phi)$ such that $Z^{\prime}$ and $c \cdot Z^{\prime}$ intersect nontrivially. It follows immediately that $\lim ^{1} \Phi$ is isomorphic to the orbit set of the action of $F$ on $Z^{\prime}$. Given $z \in Z^{\prime}$, let $\zeta$ be the restriction of $z$ to $\operatorname{GL}\left(2, \mathbf{F}_{p}\right)$. We will show that $\zeta \in Z_{\mathrm{sp}}^{1}(\Phi)$ and that the map $z \mapsto \zeta$ is injective; the proposition then follows from the obvious fact that this map respects the actions of $F$ on the objects involved.

Condition 4.41 for $\zeta$ follows from the cocycle condition 4.7. Observe that if $g \in P(i, j) \subset \mathrm{GL}\left(i+j, \mathbf{F}_{p}\right)(i+j \leq n)$, then there is an equality

$$
z(g)=e_{\#}^{i, i+j} z\left(r_{i}(g)\right) \in \Phi\left(A_{i+j}\right)
$$

which follows from the chain

$$
\begin{aligned}
z(g)=z(g) 1 & =z(g) g_{\#}\left(z\left(e^{i, i+j}\right)\right)=z\left(g e^{i, i+j}\right)=z\left(e^{i, i+j} r_{i}(g)\right) \\
& =z\left(e^{i, i+j}\right) e_{\#}^{i, i+j} z\left(r_{i}(g)\right)=e_{\#}^{i, i+j} z\left(r_{i}(g)\right) .
\end{aligned}
$$

Here we have used that $z \in Z^{\prime}$ and hence $z\left(e^{i, i+j}\right)=1$. Condition 4.42 is derived by applying 4.9 to $g \in P(1,1)$ and noting that $z(g)=1$ for $g \in \operatorname{GL}\left(1, \mathbf{F}_{p}\right)$. Let $C$ be a 1-cycle in $T\left(A_{3}\right)$ (4.3) with polarization $\left\{\alpha_{i}, \beta_{i}\right\}$ and associated difference elements $\left\{a_{i}, b_{i}\right\}$. It is clear that there is an identity

$$
a_{k} b_{k} a_{k-1} b_{k-1} \cdots b_{1} a_{1}=1
$$

in $\operatorname{GL}\left(3, \mathbf{F}_{p}\right)$. Since $a_{i} \in P(1,2)$ and $b_{i} \in P(2,1)$, it follows from 4.9 that $z\left(a_{i}\right)=1$ and $z\left(b_{i}\right)=\tilde{\zeta}\left(b_{i}\right)$ (where the notation $\tilde{\zeta}$ is from 4.4). Applying $z$ to equation 4.10 and using the cocycle property of $z$ (4.7) to expand the resulting expression now gives condition 4.43.; part of the "expansion" is actually a contraction that uses the identity

$$
a_{k} b_{k} \cdots a_{i+1} b_{i+1} a_{i}=\alpha_{1} \beta_{i}^{-1} .
$$

It remains to prove that the assignment $z \mapsto \zeta$ is injective. Suppose that $z$ and $z^{\prime}$ are two elements of $Z^{\prime}$ which agree on $\mathrm{GL}\left(2, \mathbf{F}_{p}\right)$. We will first prove by induction on $m$ that for any $2 \leq m \leq n$ the cocycles $z$ and $z^{\prime}$ agree on $\operatorname{GL}\left(i, \mathbf{F}_{p}\right)$ for all $i \leq m$. In fact, if $g \in P(i, j), i+j=m$, then $z(g)=e_{\#}^{i, m} z\left(r_{i}(g)\right)=e_{\#}^{i, m} z^{\prime}\left(r_{i}(g)\right)=z^{\prime}(g)$ by 4.9 ; thus $z$ and $z^{\prime}$ agree on a set of elements which generate $\operatorname{GL}\left(m, \mathbf{F}_{p}\right)$ (4.6), and hence agree on the whole group by the cocycle property 4.7. Suppose now that $f: A_{i} \rightarrow A_{j}$ is an arbitrary morphism in $\mathbf{A}_{n}$ with $i<j$. It is clear that there is an element $g \in \operatorname{GL}\left(j, \mathbf{F}_{p}\right)$ with $g e^{i, j}=f$, and hence

$$
z(f)=z\left(g e^{i, j}\right)=z(g) g_{\#} z\left(e^{i, j}\right)=z^{\prime}(g) g_{\#} z^{\prime}\left(e^{i, j}\right)=z^{\prime}(f) .
$$

This shows that $z$ and $z^{\prime}$ are identical. 


\section{The EXCEPtional GROUP $G_{2}$}

In this section we use 4.5 to sketch a proof of Conjecture 1.1 in the case in which $p=2$ and $\mathcal{X}$ is the 2 -completion of the exceptional compact Lie group $G_{2}$. In other words, we will prove that $\bar{\alpha}_{\mathcal{X}}$ has no nontrivial $\mathrm{H}^{*}$-clones.

As discussed in 1.2, this is part of an argument for showing that $\mathrm{B} \mathcal{X}$ is determined up to homotopy by $\mathrm{H}^{*} \mathrm{~B} \mathcal{X}$ (as an algebra over the Steenrod algebra). Beyond what we do in this section, there are three steps necessary to complete this uniqueness argument:

1. Show that for each object $v$ of $\mathbf{A}_{\mathcal{X}}$ the space $\bar{\alpha}_{\mathcal{X}}(v)$ is determined up to homotopy by its cohomology.

2. Show that for each object $v$ of $\mathbf{A}_{\mathcal{X}}$, appropriate automorphisms of $\mathrm{H}^{*} \bar{\alpha}_{\mathcal{X}}(v)$ are realized by self-equivalences of $\bar{\alpha} \mathcal{X}(v)$.

3. Show that $\bar{\alpha}_{\mathcal{X}}$ has up to the relevant notion of equivalence only one lift to a functor $\mathbf{A}_{\mathcal{X}} \rightarrow \mathbf{S p}$.

The authors intend to carry out these steps in a future paper.

Suppose that $G$ is a compact Lie group (eventually $G_{2}$ ). Let $\mathbf{A}_{G}$ be the category whose objects are the non-trivial elementary abelian subgroups of $G$; a morphism $V \rightarrow V^{\prime}$ in $\mathbf{A}_{G}$ is a monomorphism $f: V \rightarrow V^{\prime}$ of abelian groups with the property that there exists an element $g \in G$ such that $f(x)=g x g^{-1}$ for all $x \in V$. As in [13], there is a functor $\alpha_{G}^{\mathrm{op}}: \mathbf{A}_{G} \rightarrow \mathbf{S p}$ which sends $V$ to the Borel construction $E G \times_{G}\left(G / C_{G}(V)\right)$, where $C_{G}(V)$ is the centralizer of $V$ in $G$. (Note that this Borel construction has the homotopy type of the classifying space $\mathrm{B} C_{G}(V)$.)

5.1 Remark. The effect on a morphism $f: V \rightarrow V^{\prime}$ of $\alpha_{G}$ is as follows. Let $g \in G$ be an element such that $f(x)=g x g^{-1}$ for all $x \in V$. The map $h \mapsto g^{-1} h g$ gives a homomorphism $C_{G}\left(V^{\prime}\right) \rightarrow C_{G}(V)$, and so there is a $G$-equivariant map $G / C_{G}\left(V^{\prime}\right) \rightarrow G / C_{G}(V)$ given by $x C_{G}\left(V^{\prime}\right) \mapsto x g C_{G}(V)$. The induced map of Borel constructions is $\alpha_{G}(f)$. It does not depend upon the choice of the element $g$.

The following proposition is well-known; it is a consequence of $[9,2.2],[12$, proof of 8.1], and the fact that if $G$ is a compact Lie group with $\pi_{0} G$ a $p$-group, and $V$ is an elementary abelian $p$-group, then there is a natural weak equivalence $\operatorname{Map}(\mathrm{B} V, \mathrm{~B} G) \hat{p} \rightarrow \operatorname{Map}\left(\mathrm{B} V, \mathrm{~B} G_{p}^{\hat{p}}\right)[7,4.5]$.

5.2 Proposition. Suppose that $G$ is a compact Lie group such that $\pi_{0} G$ is a pgroup, and let $\mathcal{X}$ be the p-compact group $G_{p}$. Then there is an equivalence of categories e $: \mathbf{A}_{G} \rightarrow \mathbf{A}_{\mathcal{X}}$ such that the composite functor $\alpha_{\mathcal{X}} \cdot e$ is weakly equivalent to $\left(\alpha_{G}\right) \hat{p}$.

Remark. A weak equivalence between two functors $\alpha, \alpha^{\prime}: \mathbf{A}_{G} \rightarrow \mathbf{S p}$ is a natural transformation which gives an ordinary weak equivalence of spaces for each object of $\mathbf{A}_{G}$ (cf. 6.1). The functors $\alpha$ and $\alpha^{\prime}$ are weakly equivalent if they are connected by a zigzag of weak equivalences.

We will need to deal with certain elements and subgroups of $\mathrm{SO}(4)$ and of $G_{2}$. Let $\tau_{1}$ denote the central diagonal matrix $\operatorname{diag}(-1,-1,-1,-1)$ in $\mathrm{SO}(4), \tau_{2}$ the matrix $\operatorname{diag}(-1,-1,1,1)$ and $\tau_{3}$ the block matrix

$$
\tau_{3}=\left(\begin{array}{cc}
N & 0 \\
0 & N
\end{array}\right) \quad \text { with } \quad N=\left(\begin{array}{cc}
0 & 1 \\
1 & 0
\end{array}\right)
$$


These matrices generate a subgroup of $\mathrm{SO}(4)$ isomorphic to $(\mathbf{Z} / 2)^{3}$. The symbol $T^{2}$ will denote the maximal torus of $\mathrm{SO}(4)$ given by block matrices

$$
\left(\begin{array}{cc}
M_{1} & 0 \\
0 & M_{2}
\end{array}\right) \text { with } \quad M_{i}=\left(\begin{array}{cc}
\cos \theta_{i} & -\sin \theta_{i} \\
\sin \theta_{i} & \cos \theta_{i}
\end{array}\right)
$$

and $T_{\text {ext }}^{2} \supset T^{2}$ the subgroup of $\mathrm{SO}(4)$ generated by $T^{2}$ and $\tau_{3}$.

Let $V_{1}$ be some fixed subgroup of $G_{2}$ isomorphic to $\mathbf{Z} / 2$; according to [14, 2.3], such a subgroup is unique up to conjugacy, and the centralizer $C_{G_{2}}\left(V_{1}\right)$ is isomorphic to $\mathrm{SO}(4)$. Fix an isomorphism $C_{G_{2}}\left(V_{1}\right) \cong \mathrm{SO}(4)$. It is clear that under this isomorphism $V_{1}$ is identified with the center $\left\langle\tau_{1}\right\rangle$ of $\mathrm{SO}(4)$. Let $V_{2} \subset \mathrm{SO}(4) \subset G_{2}$ be the subgroup $\left\langle\tau_{1}, \tau_{2}\right\rangle$ and $V_{3}$ the subgroup $\left\langle\tau_{1}, \tau_{2}, \tau_{3}\right\rangle$.

If $A$ is a subgroup of $G_{2}$ let $N_{G_{2}}(A)$ denote its normalizer; note that the quotient $N_{G_{2}}(A) / C_{G_{2}}(A)$ acts faithfully on $A$ by conjugation.

5.3 Proposition. For each integer $i$ with $1 \leq i \leq 3$, the subgroup $V_{i} \subset G_{2}$ described above is up to conjugacy the unique subgroup of $G_{2}$ isomorphic to $(\mathbf{Z} / 2)^{i}$. There is no subgroup of $G_{2}$ isomorphic to $(\mathbf{Z} / 2)^{4}$. The group $C_{G_{2}}\left(V_{1}\right)$ is isomorphic to $\mathrm{SO}(4)$, the group $C_{G_{2}}\left(V_{2}\right)$ is isomorphic to $T_{\mathrm{ext}}^{2}$, and the group $C_{G_{2}}\left(V_{3}\right)$ to $V_{3}$ itself. For $1 \leq i \leq 3$ the conjugation action gives an isomorphism

$$
N_{G_{2}}\left(V_{i}\right) / C_{G_{2}}\left(V_{i}\right) \cong \operatorname{Aut}\left(V_{i}\right) \cong \mathrm{GL}\left(i, \mathbf{F}_{2}\right) .
$$

Proof. The required properties of $V_{1}$ and $V_{3}$ are given in [14, 2.3]. As far as $V_{2}$ is concerned, note that, by uniqueness of $V_{1}$ up to conjugacy and the isomorphism $C_{G_{2}}\left(V_{1}\right) \cong \mathrm{SO}(4)$, the conjugacy classes of homomorphisms $\mathbf{Z} / 2 \times \mathbf{Z} / 2 \rightarrow G_{2}$ correspond bijectively to conjugacy classes in $\mathrm{SO}(4)$ of non-central involutions; by linear algebra there is only one such class, the conjugacy class of $\tau_{2}$. The centralizer of $\tau_{2}$ in $\mathrm{SO}(4)$, which is the same as the centralizer of $\left\langle\tau_{1}, \tau_{2}\right\rangle$ in $G_{2}$, is $T_{\text {ext }}^{2}$. Since there is only one conjugacy class of homomorphism $\mathbf{Z} / 2 \times \mathbf{Z} / 2 \rightarrow G_{2}$, every automorphism of $\left\langle\tau_{1}, \tau_{2}\right\rangle$ must be realized by conjugation with an element of $G_{2}$; this gives the isomorphism $N_{G_{2}}\left(V_{2}\right) / C_{G_{2}}\left(V_{2}\right) \cong \mathrm{GL}\left(2, \mathbf{F}_{2}\right)$. Finally, again by uniqueness of $V_{1}$, any $(\mathbf{Z} / 2)^{4}$ in $G_{2}$ would give $(\mathbf{Z} / 2)^{4}$ in $\mathrm{SO}(4)$, and such a subgroup of $\mathrm{SO}(4)$ does not exist.

Now let $\mathbf{C}$ be the full subcategory of $\mathbf{A}_{G_{2}}$ generated by the elementary abelian 2-subgroups $V_{1}=\left\langle\tau_{1}\right\rangle, V_{2}=\left\langle\tau_{1}, \tau_{2}\right\rangle$ and $V_{3}=\left\langle\tau_{1}, \tau_{2}, \tau_{3}\right\rangle$ of $G_{2}$, and let $\mathbf{A}_{3}$ be the category of $\S 4$. The unique basis-preserving vector space isomorphisms $V_{i} \cong A_{i}$ give a functor $\iota: \mathbf{C} \rightarrow \mathbf{A}_{3}$ which by 5.3 is an isomorphism of categories. Since $\mathbf{C}$ is a skeletal subcategory of $\mathbf{A}_{G_{2}}$ (i.e., a full subcategory which contains one object of each isomorphism type) the composite of $\iota^{-1}: \mathbf{A}_{3} \rightarrow \mathbf{C}$ with the inclusion $\mathbf{C} \rightarrow \mathbf{A}_{G_{2}}$ is an equivalence of categories. Let $\alpha: \mathbf{A}^{\mathrm{op}} \rightarrow \mathbf{S p}$ be the restriction of $\alpha_{G_{2}}$ to $\mathbf{A}_{3}, \hat{\alpha}$ the 2-completion of $\alpha$, and $F, \hat{F}$ the diagrams in Ho underlying $\alpha$, $\hat{\alpha}$ respectively.

By 5.2, the following proposition is equivalent to the result stated at the beginning of this section.

5.4 Proposition. The functor $\hat{F}: \mathbf{A}^{\mathrm{op}} \rightarrow$ Ho has no $\mathrm{H}^{*}$-clones.

5.5 Notation. If $G$ is a compact Lie group, we will let $\hat{\mathrm{B}} G$ denote $\mathrm{B} G_{2}$. The symbol $\mathbf{Z}_{2}$ will stand for the ring of 2-adic integers, and $\Gamma_{i}(n)$ for the subgroup of $\operatorname{GL}\left(n, \mathbf{Z}_{2}\right)$ consisting of matrices which are congruent to the identity matrix mod $2^{i}$. 
Proof of 5.4. Since the functor $\hat{F}$ is $\mathrm{H}^{*}$-centric up to homotopy (3.1), the proposition is equivalent to the assertion that $\lim ^{1} \phi_{\hat{F}}$ is trivial.

The functor $\hat{\alpha}$ has the following properties:

1. $\hat{\alpha}\left(A_{1}\right) \sim \hat{\mathrm{B} S O}(4), \hat{\alpha}\left(A_{2}\right) \sim \hat{\mathrm{B}} T_{\mathrm{ext}}^{2}$, and $\hat{\alpha}\left(A_{3}\right) \sim \hat{\mathrm{B}} A_{3}$.

2. The map $\hat{\alpha}\left(e^{1}\right): \hat{\alpha}\left(A_{2}\right) \rightarrow \hat{\alpha}\left(A_{1}\right)$ is up to homotopy the map $\hat{\mathrm{B}} T_{\text {ext }}^{2} \rightarrow$ $\hat{\mathrm{B} S O}(4)$ induced by the inclusion $T_{\text {ext }}^{2} \subset \mathrm{SO}(4)$.

To study the functor $\phi_{\hat{F}}$ it is necessary to calculate the groups Aut ${ }_{i}$ of homotopy classes of homotopy self-equivalences of $\hat{\alpha}\left(A_{i}\right), i=1,2,3$, and then determine the subgroups $\phi_{\hat{F}}\left(A_{i}\right)$ of equivalences which induce the identity on $\mathrm{H}^{*}$. Picking out the groups $\phi_{\hat{F}}\left(A_{i}\right)$ turns out to be relatively easy, because for $i=1,2,3$ the restriction map $\mathrm{H}^{*} \hat{\alpha}\left(A_{i}\right) \rightarrow \mathrm{H}^{*} \mathrm{~B} V_{3}$ is a monomorphism. The case $i=1$ of this monomorphism assertion is classical, the case $i=3$ is trivial, and the case $i=2$ is proved either by direct calculation or by combining the exactness of Lannes' functor $T$ with the case $i=1[15,2.1$, p. 203].

Let $L$ denote the $\mathbf{Z}_{2}$ module given by $\pi_{2} \hat{\mathrm{B}} T^{2}$. The group $V_{2}$ is the subgroup of elements of exponent 2 in $T^{2}$, and so the long exact homotopy sequence associated to the fibration sequence

$$
\mathrm{B} V_{2} \rightarrow \hat{\mathrm{B}} T^{2} \stackrel{\hat{\mathrm{B}}\left(t \mapsto t^{2}\right)}{\longrightarrow} \hat{\mathrm{B}} T^{2}
$$

gives a natural isomorphism

$$
V_{2} \cong \mathbf{Z} / 2 \otimes L
$$

Give $L$ the standard basis $\left\{l_{1}, l_{2}\right\}$ which comes from the isomorphism $T^{2} \cong \mathrm{SO}(2) \times$ $\mathrm{SO}(2) \subset \mathrm{SO}(4)$ and choice of a generator for $\pi_{1} \mathrm{SO}(2)$. In this basis the conjugation image of the Weyl group of $\mathrm{SO}(4)$ in $\operatorname{Aut}(L)$ is the rank 2 elementary abelian group generated by the matrices

$$
\pm\left(\begin{array}{ll}
0 & 1 \\
1 & 0
\end{array}\right) .
$$

Now set $\ell_{1}=l_{1}+l_{2}$ and $\ell_{2}=l_{1}$. In this new basis the conjugation image of the Weyl group of $\mathrm{SO}(4)$ in $\operatorname{Aut}(L)$ is the subgroup $W_{1}$ of $\mathrm{GL}\left(2, \mathbf{Z}_{2}\right)$ generated by the matrices

$$
\pm\left(\begin{array}{cc}
1 & 1 \\
0 & -1
\end{array}\right)
$$

(see $[10, \S 3]$ for a similar calculation). Moreover, the basis $\left\{\ell_{1}, \ell_{2}\right\}$ is adapted to the basis $\left\{\tau_{1}, \tau_{2}\right\}$ of $V_{2}$, in the sense that the reduction mod $2(5.6)$ of $\ell_{i}$ is $\tau_{i}$.

Let $N_{1}$ denote the normalizer of $W_{1}$ in $\operatorname{GL}\left(2, \mathbf{Z}_{2}\right)$. By $[10, \S 5]$ there is a natural isomorphism Aut $1 \cong N_{1} / W_{1}$. The group $N_{1} / W_{1}$ is itself isomorphic to the wreath product $\Sigma_{2} 2 \Gamma_{2}(1)[10,5.4$, proof of 5.5]. (Note in checking this reference that the obvious map $\Gamma_{2}(1) \rightarrow \operatorname{GL}\left(1, \mathbf{Z}_{2}\right) /\langle \pm 1\rangle$ is an isomorphism.) It follows easily that the subgroup $\phi_{\hat{F}}\left(A_{1}\right)$ of Aut $_{1}$ is the kernel $\Gamma_{2}(1) \times \Gamma_{2}(1)$ of the projection Aut $_{1} \rightarrow \Sigma_{2}$. The inclusion of this product into $N_{1} / W_{1}$ is induced by the map $\Gamma_{2}(1) \times \Gamma_{2}(1) \rightarrow \Gamma_{1}(2)$ given by the formula

$$
(u, v) \mapsto\left(\begin{array}{cc}
u & (u-v) / 2 \\
0 & v
\end{array}\right) .
$$


Let $W_{2}$ denote the central subgroup of $\mathrm{GL}\left(2, \mathbf{Z}_{2}\right)$ generated by the negative of the identity matrix and $N_{2}=\mathrm{GL}\left(2, \mathbf{Z}_{2}\right)$ its normalizer. An analysis along the lines of $[10, \S 5]$ but substantially more elementary shows that there is a natural isomorphism Aut $2 \cong N_{2} / W_{2}$. Denote this quotient by $\overline{\mathrm{GL}}\left(2, \mathbf{Z}_{2}\right)$. The group $\phi_{\hat{F}}\left(A_{2}\right)$ is the image $\bar{\Gamma}_{1}(2)$ in $\overline{G L}\left(2, \mathbf{Z}_{2}\right)$ of $\Gamma_{1}(2)$.

It is clear that $\mathrm{Aut}_{3}$ is isomorphic to $\mathrm{GL}\left(3, \mathbf{F}_{2}\right)$ and that its subgroup $\phi_{\hat{F}}\left(A_{3}\right)$ is trivial.

The next step is to obtain information about the maps in the diagram $\phi_{\hat{F}}$. A naturality argument shows that the map $\phi_{\hat{F}}\left(e^{1}\right): \Gamma_{2}(1) \times \Gamma_{2}(1) \rightarrow \bar{\Gamma}_{1}(2)$ is induced by formula 5.7. The action of $\operatorname{Aut}\left(A_{2}\right)$ on $\hat{F}\left(A_{2}\right)$ is induced by the conjugation action of $N_{G_{2}}\left(V_{2}\right) / C_{G_{2}}\left(V_{2}\right)$ on $\mathrm{B} C_{G_{2}}\left(V_{2}\right)$; homotopically this conjugation action is the one provided by the fact that $\mathrm{B} C_{G_{2}}\left(V_{2}\right)$ is a regular covering space of $\mathrm{B} N_{G_{2}}\left(V_{2}\right)$ with covering group $N_{G_{2}}\left(V_{2}\right) / C_{G_{2}}\left(V_{2}\right)$. From this it follows that the map $f: \operatorname{Aut}\left(A_{2}\right) \rightarrow$ Aut $_{2} \cong \overline{\mathrm{GL}}\left(2, \mathbf{Z}_{2}\right)$ given by $\hat{F}$ induces an isomorphism $\operatorname{Aut}\left(A_{2}\right) \cong \mathrm{GL}\left(2, \mathbf{F}_{2}\right)$ upon reducing mod 2. Since the basis $\left\{\ell_{1}, \ell_{2}\right\}$ used in making the identification $\mathrm{Aut}_{2} \cong \overline{\mathrm{GL}}\left(2, \mathbf{Z}_{2}\right)$ is adapted to the basis $\left\{\tau_{1}, \tau_{2}\right\}$ for $L$, this isomorphism $\operatorname{Aut}\left(A_{2}\right) \cong \mathrm{GL}\left(2, \mathbf{F}_{2}\right)$ is actually the identity map. The action of $\operatorname{Aut}\left(A_{2}\right)$ on $\bar{\Gamma}_{1}(2)$ given by $\phi_{\hat{F}}$ is obtained by letting $\operatorname{Aut}\left(A_{2}\right)$ act on $\bar{\Gamma}_{1}(2)$ by conjugation via the homomorphism $f$.

Denote by $\operatorname{gl}\left(2, \mathbf{F}_{2}\right)$ the vector space of $2 \times 2$ matrices over $\mathbf{F}_{2}$, and by $\operatorname{pgl}\left(2, \mathbf{F}_{2}\right)$ its quotient by the subgroup generated by the identity matrix. Matrix conjugation gives actions of $\operatorname{Aut}\left(A_{2}\right)=\mathrm{GL}\left(2, \mathbf{F}_{2}\right)$ on both $\operatorname{gl}\left(2, \mathbf{F}_{2}\right)$ and $\operatorname{pgl}\left(2, \mathbf{F}_{2}\right)$. Let $\bar{\Gamma}_{i}(2)$ be the image in $\bar{\Gamma}_{1}(2)$ of $\Gamma_{i}(2)$. By the discussion in the last paragraph there are $\operatorname{Aut}\left(A_{2}\right)$-equivariant isomorphisms

$$
\bar{\Gamma}_{i}(2) / \bar{\Gamma}_{i+1}(2) \cong \begin{cases}\operatorname{pgl}\left(2, \mathbf{F}_{2}\right), & i=1 \\ \Gamma_{i}(2) / \Gamma_{i+1}(2) \cong \operatorname{gl}\left(2, \mathbf{F}_{2}\right), & i>1\end{cases}
$$

Consider now an element $\zeta \in Z_{\mathrm{sp}}^{1}\left(\phi_{\hat{F}}\right)(\S 4) ; \zeta$ is a function $\operatorname{GL}\left(2, \mathbf{F}_{2}\right) \rightarrow \bar{\Gamma}_{1}(2)$ which satisfies conditions 1 and 2 of 4.4. (Note that condition 4.4(3) is automatically satisfied, because $\phi_{\hat{F}}\left(A_{3}\right)$ is the trivial group.) Consider the elements of $\mathrm{GL}\left(2, \mathbf{F}_{2}\right)$ given by the matrices

$$
s=\left(\begin{array}{ll}
1 & 1 \\
1 & 0
\end{array}\right) \quad \text { and } \quad t=\left(\begin{array}{ll}
1 & 1 \\
0 & 1
\end{array}\right)
$$

These generate $\operatorname{GL}\left(2, \mathbf{F}_{2}\right)$ subject to the relations $s^{3}=1, t^{2}=1, t s t=s^{2}$. Since $\zeta(t)=1$, the cocycle $\zeta$ is determined by $\zeta(s)$. The idea of the argument is now to gradually deform $\zeta$ toward the trivial cocycle, and use the completeness of $\mathbf{Z}_{2}$ to pass to the limit. More precisely, we will inductively construct elements $w_{i} \in \Gamma_{i+1}(1) \times$ $\Gamma_{i+1}(1)$ such that if $x_{i}=w_{i} \cdots w_{1}$ and $y_{i}=e_{\#}^{1}\left(x_{i}\right)$, then $y_{i} \zeta(s) s_{\#}\left(y_{i}\right)^{-1} \in \Gamma_{i+1}(2)$. The sequence $\left\{x_{i}\right\}$ then converges in the 2 -adic topology on $\Gamma_{2}(1) \times \Gamma_{2}(1)$, and its limit $x$ gives a trivialization of the cocycle $\zeta$.

The construction of $w_{1}$ is left to the reader since it is very similar to the inductive step we are about to describe. Assume that suitable $w_{i}$, together with the associated elements $x_{i}$ and $y_{i}$, have been chosen for $i<n(n>1)$. Let $\xi \in Z_{\mathrm{sp}}^{1}\left(\phi_{\hat{F}}\right)$ be determined by $\xi(s)=y_{n-1} \zeta(s) s_{\#}\left(y_{n-1}\right)^{-1}$, and let $\bar{\xi}: \operatorname{GL}\left(2, \mathbf{F}_{2}\right) \rightarrow \operatorname{gl}\left(2, \mathbf{F}_{2}\right)$ be 
the reduction of $\xi$ modulo $\Gamma_{n+1}(2)$. With the group operation in $\operatorname{gl}\left(2, \mathbf{F}_{2}\right)$ written additively, $\bar{\xi}$ satisfies the cocycle condition

$$
\bar{\xi}(g h)=\bar{\xi}(g)+g_{\#} \bar{\xi}(h), \quad g, h \in \mathrm{GL}\left(2, \mathbf{F}_{2}\right) .
$$

Here, as noted above, the action of $g$ on $\bar{\xi}(h)$ is by conjugation. Expanding the left hand side of the equation $\bar{\xi}\left(s^{3}\right)=0$ with 5.8 gives $\bar{\xi}(s)+s_{\#} \bar{\xi}(s)+s_{\#}^{2} \bar{\xi}(s)=0$, which by explicit calculation implies that $\bar{\xi}(s)$ has the form

$$
\left(\begin{array}{cc}
b+c & b \\
c & b+c
\end{array}\right)
$$

Expanding both sides of $\bar{\xi}(t s t)=\bar{\xi}\left(s^{2}\right)$ by the same technique gives

$$
\left(\begin{array}{cc}
b & b+c \\
c & b
\end{array}\right)=t_{\#} \bar{\xi}(s)=\bar{\xi}(s)+s_{\#} \bar{\xi}(s)=\left(\begin{array}{cc}
b & c \\
b+c & b
\end{array}\right)
$$

which implies $b=0$. If $c=0$ let $w_{n}$ be the identity element, otherwise choose $w_{n}=(u, v) \in \Gamma_{n+1}(1) \times \Gamma_{n+1}(1)$ such that $(u-v) / 2$ is not congruent to zero $\bmod 2^{n}$. If $M$ is the reduction $\bmod \Gamma_{n+1}(2)$ of $e_{\#}^{1}\left(w_{n}\right)$, then (5.7)

$$
M+\bar{\xi}(s)+s_{\#}(M)=\left(\begin{array}{ll}
0 & 1 \\
0 & 0
\end{array}\right)+\left(\begin{array}{ll}
1 & 0 \\
1 & 1
\end{array}\right)+\left(\begin{array}{ll}
1 & 1 \\
1 & 1
\end{array}\right)=0
$$

This shows that $w_{n}$ has the required inductive property.

\section{The COHOMOLOGY CATEGORY}

For convenience of exposition, in this section "space" means "simplicial set". Let CoHo denote the category whose objects are spaces and whose morphisms are cohomology classes of maps; more formally, CoHo is the quotient category of $\mathbf{H o}$ in which two maps $f, g: X \rightarrow Y$ are considered equivalent if they induce the same map $\mathrm{H}^{*} Y \rightarrow \mathrm{H}^{*} X$. Let $\pi: \mathbf{S p} \rightarrow \mathbf{C o H o}$ be the obvious functor.

6.1 Definition. Suppose that $\bar{X}: \mathbf{D} \rightarrow \mathbf{C o H o}$ is a functor. A realization of $\bar{X}$ is a pair $(X, s)$, where $X: \mathbf{D} \rightarrow \mathbf{S p}$ is a functor and $s: \pi X \rightarrow \bar{X}$ is a natural equivalence. A weak equivalence $t:(X, s) \rightarrow\left(X^{\prime}, s^{\prime}\right)$ between two such realizations is a natural transformation from $X$ to $X^{\prime}$ such that

1. for each object $d$ of $\mathbf{D}, t_{d}: X(d) \rightarrow X^{\prime}(d)$ is a weak equivalence of spaces, and

2. the composite natural transformation $s^{\prime} \cdot \pi(t)$ is equal to $s$.

The realization complex $r \bar{X}$ of $\bar{X}$ is defined to be the nerve of the category whose objects are the realizations of $\bar{X}$ and whose morphisms are the weak equivalences between these realizations.

The aim of this section is to calculate the homotopy type of $r \bar{X}$ for diagrams $\bar{X}$ which simultaneously satisfy the condition of $\S 2$ and the "centric" condition of [7]. The main result, which requires some preparation to state, is thus a combination of 2.4 and the results in [7]. Our motivation for looking at this is Conjecture 6.8, which essentially states that for a $p$-compact group $\mathcal{X}$, the homotopy type of $\mathrm{B} \mathcal{X}$ 
should be uniquely determined by the decomposition diagram $\alpha_{\mathcal{X}}$ considered as a diagram in the cohomology category. The main result of this section provides a mechanism for verifying this conjecture in particular cases.

Remark. The realization complex $r \bar{X}$ of 6.1 is the nerve of a category which is not small. Nevertheless this nerve is homotopically small [4] and it is possible to make standard homotopy theoretic constructions with it. The components of $r \bar{X}$ are in 1-1 correspondence with weak equivalence classes $\{(X, s)\}$ of realizations of $\bar{X}$, and the component corresponding to a particular $(X, s)$ is equivalent to the classifying space of an appropriate complex $[4,3.5]$ of homotopy automorphisms of $X$ (in our situation this is the complex of homotopy automorphisms of $X$ which for each object $d$ of $\mathbf{D}$ induce the identity automorphism of $\left.\mathrm{H}^{*} X(d)\right)$.

6.2 Remark. Let $\bar{X}: \mathbf{D} \rightarrow \mathbf{C o H o}$ be a functor, $\mathbf{D}^{\prime}$ a small category, and $F: \mathbf{D}^{\prime} \rightarrow$ $\mathbf{D}$ a functor. Let $F^{*} \bar{X}: \mathbf{D}^{\prime} \rightarrow \mathbf{C o H o}$ be the composite of $\bar{X}$ with $F$. It is easy to see that $F$ induces a natural map $r \bar{X} \rightarrow r\left(F^{*} \bar{X}\right)$.

If $f: A \rightarrow B$ is a map in $\mathbf{S p}$ or $\mathbf{C o H o}$, let $\operatorname{Map}(A, B)_{\{f\}}$ denote the subspace of the mapping space $\operatorname{Map}(A, B)$ consisting of maps $f^{\prime}$ such that $\mathrm{H}^{*}\left(f^{\prime}\right)=\mathrm{H}^{*}(f)$.

6.3 Definition. A map $f: A \rightarrow B$ between fibrant simplicial sets is said to be $\mathrm{H}^{*}$-centric if

1. $[A, A]_{\{\mathrm{id}\}}=\pi_{0} \operatorname{Map}(A, A)_{\{\mathrm{id}\}}$ is a group under composition, and

2. composition with $f$ induces a weak equivalence

$$
\operatorname{Map}(A, A)_{\{\mathrm{id}\}} \stackrel{\simeq}{\longrightarrow} \operatorname{Map}(A, B)_{\{f\}} .
$$

A map $f$ in $\mathbf{C o H o}$ is said to be $\mathrm{H}^{*}$-centric if any representative of $f$ is $\mathrm{H}^{*}$-centric (equivalently, if all representatives are $\mathrm{H}^{*}$-centric). A functor $F: \mathbf{D} \rightarrow \mathbf{C o H o}$ is said to be $\mathrm{H}^{*}$-centric if $F(g)$ is $\mathrm{H}^{*}$-centric for each morphism $g$ of $\mathbf{D}$.

Remark. It is clear that a map $f: A \rightarrow B$ is $\mathrm{H}^{*}$-centric if and only if $f$ is both $\mathrm{H}^{*}$-centric up to homotopy in the sense of $\S 2$ and centric in the sense of [7]. If $\mathcal{X}$ is a $p$-compact group, then the diagram in CoHo underlying $\alpha_{\mathcal{X}}$ is $\mathrm{H}^{*}$-centric up to homotopy by 3.1. It is possible to show that this diagram is centric in the sense of [7] by combining $[11,8.2]$ with the fibration argument in the proof of $[7,4.2]$. See also $[12,11.15]$. Note that when it comes to dealing with $p$-compact groups, as opposed to compact Lie groups, all of the worries about $p$-completion in $[7, \S 4]$ become irrelevant and the argument significantly shrinks.

6.4 Remark. If $A$ is a fibrant simplicial set let $\mathrm{h}(A) \operatorname{denote} \operatorname{Map}(A, A)_{\{\text {id }\}}$. Suppose that $f: A \rightarrow B$ is a map in CoHo, let $M=\operatorname{Map}(A, B)_{\{f\}}, G=\mathrm{h}(A)$ and $H=$ $\mathrm{h}(B)$. Let ${ }_{G} M$ denote the Borel construction of the right action of $G$ on $M, M_{H}$ the Borel construction of the left action of $H$ on $M$, and ${ }_{G} M_{H}$ the corresponding double Borel construction (which is the Borel construction of the right action of $G$ on $M_{H}$ or equivalently the Borel construction of the left action of $H$ on ${ }_{G} M$ ). There are fibration sequences

$$
\begin{gathered}
M \rightarrow_{G} M \rightarrow \mathrm{B} G, \\
{ }_{G} M \rightarrow_{G} M_{H} \rightarrow \mathrm{B} H, \\
M_{H} \rightarrow{ }_{G} M_{H} \rightarrow \mathrm{B} G .
\end{gathered}
$$


Suppose that $f$ is $\mathrm{H}^{*}$-centric; this implies that ${ }_{G} M$ is weakly contractible and hence that ${ }_{G} M_{H} \rightarrow \mathrm{BH}$ is an equivalence, so that the third fibration sequence above determines up to homotopy a map $\mathrm{B} H \rightarrow \mathrm{B} G$. We will denote this map $f^{b}$. The construction of $f^{b}$ is parallel to what was done in $\S 2$ using 2.3 to construct $\phi_{F}(g)$. We will show below that this construction can be rigidified in a natural way; this rigidification gives, for any $\mathrm{H}^{*}$-centric diagram $\bar{X}$, an associated diagram in $\mathbf{S p}$ whose constituent spaces are equivalent to classifying spaces of self-equivalences of the spaces $\bar{X}(d)$.

For any object $d$ of $\mathbf{D}$, let $\mathbf{D} \downarrow d$ denote the over category [1, XI, §2] [16, p. 46] of the identity functor. The objects of this category are pairs $\left(d^{\prime}, g\right)$ where $d^{\prime}$ is an object of $\mathbf{D}$ and $f: d^{\prime} \rightarrow d$ is a map; a morphism $\left(d^{\prime}, f\right) \rightarrow\left(d^{\prime \prime}, g\right)$ is a map $h: d^{\prime} \rightarrow d^{\prime \prime}$ such that $g h=f$. There is a forgetful functor $O_{d}: \mathbf{D} \downarrow d \rightarrow \mathbf{D}$ which sends a pair $(d, f)$ to $d$. Given a diagram $\bar{X}: \mathbf{D} \rightarrow \mathbf{C o H o}$, there is an induced diagram

$$
O_{d}^{*} \bar{X}=\bar{X} \cdot O_{d}: \mathbf{D} \downarrow d \rightarrow \mathbf{C o H o}
$$

for each object $d$ of $\mathbf{D}$, as well as (6.2) a functor $r_{\mathbf{D}^{\text {op }}} \bar{X}: \mathbf{D}^{\text {op }} \rightarrow \mathbf{S p}$ which maps an object $d \in \mathbf{D}^{\text {op }}$ to the realization complex $r\left(O_{d}^{*} \bar{X}\right)$.

As in [7, p. 577], in order to form homotopy limits [1] which have homotopy invariant meaning we sometimes have to replace a given functor $Y: \mathbf{D} \rightarrow \mathbf{S p}$ by a fibrant one $Y^{\prime}$, i.e., by a weakly equivalent functor $Y^{\prime}$ such that $Y^{\prime}(d)$ is a Kan complex for each object $d$ of $\mathbf{D}$. We will write $Y^{f}$ to denote such a replacement. The following theorem is proved in exactly the same way as $[7,2.1]$; it is a derivative form of the basic diagram classification result in [3].

6.5 Theorem. Suppose that $\mathbf{D}$ is a small category and that $\bar{X}: \mathbf{D} \rightarrow \mathbf{C o H o}$ is a functor. Then the natural map

$$
r \bar{X}=\lim r_{\mathbf{D}^{\mathrm{op}}} \bar{X} \rightarrow \operatorname{holim}\left(r_{\mathbf{D}^{\mathrm{op}}} \bar{X}\right)^{f}
$$

is a weak equivalence.

We have now come to the main result of this section, which states that for a diagram $\bar{X}$ with shape $\mathbf{D}$ which is $\mathrm{H}^{*}$-centric, the functor $r_{\mathbf{D}^{\mathrm{op}}} \bar{X}$ is the rigidification promised in 6.4. Note that Theorem 6.5 guarantees that the homotopy limit of this rigidification is equivalent to $r \bar{X}$.

6.6 Theorem. Suppose that $\mathbf{D}$ is a small category and that $\bar{X}: \mathbf{D} \rightarrow \mathbf{C o H o}$ is a functor which is $\mathrm{H}^{*}$-centric. Then for each object $d$ of $\mathbf{D}$ the space $r_{\mathbf{D}^{\circ \mathrm{p}}} \bar{X}$ is weakly homotopy equivalent in a natural way to $\operatorname{Bh}(\bar{X}(d))$. Under these equivalences, for each $g: d \rightarrow d^{\prime}$ in $\mathbf{D}$ the map $r_{\mathbf{D}^{\mathrm{op}}} \bar{X}(g)$ is homotopic to the map

$$
\bar{X}(g)^{b}: \operatorname{Bh}\left(\bar{X}\left(d^{\prime}\right)\right) \rightarrow \operatorname{Bh}(\bar{X}(d)) .
$$

described in 6.4.

Proof. This is essentially the same as the proof of $[7,2.2]$. The main issue (cf. the proof of $[7,2.3])$ is to show that if

$$
A_{0} \stackrel{f_{1}}{\longrightarrow} A_{1} \stackrel{f_{2}}{\longrightarrow} \cdots \stackrel{f_{n}}{\longrightarrow} A_{n}
$$


is a chain of maps in CoHo such that each $f_{i}$ is $\mathrm{H}^{*}$-centric, then the natural projection

$$
\mathrm{B}\left(\mathrm{h}\left(A_{0}\right), \operatorname{Map}\left(A_{0}, A_{1}\right)_{\left\{f_{1}\right\}}, \mathrm{h}\left(A_{1}\right), \ldots, \operatorname{Map}\left(A_{n-1}, A_{n}\right)_{\left\{f_{n}\right\}}, \mathrm{h}\left(A_{n}\right)\right)
$$

is an equivalence; the iterated bar construction on the left here is described in [5, 9.1]. This is proved by an induction [5, 9.2(vii)] that depends only on the observation made in 6.4 about the contractibility of certain ordinary Borel constructions. $\square$

Let Grpd denote the category of groupoids, so that Grp is the full subcategory of Grpd consisting of groupoids with a single object.

6.7 Definition. If $H, H^{\prime}: \mathbf{D} \rightarrow$ Grpd are functors, a natural transformation $t$ : $H \rightarrow H^{\prime}$ is said to be a weak equivalence if $t_{d}$ is an equivalence of categories for each object $d \in \mathbf{D}$.

Let $\mathbf{D}$ and $\bar{X}$ be as in 6.6 , let $\Pi_{1}$ be the composite of $r_{\mathbf{D}^{\text {op }}} \bar{X}$ with the fundamental groupoid functor, and let $\mathrm{B} \Pi_{1}$ be the further composite with the classifying space functor. It is possible to check that finding a point in holim $\left(B \Pi_{1}\right)$ is equivalent to lifting $\bar{X}$ to a functor $F: \mathbf{D} \rightarrow \mathbf{H o}$ or, from another point of view, to finding a weak equivalence (6.7) between $\Pi_{1}$ and a diagram of groups. If holim $\left(B \Pi_{1}\right)$ is nonempty, i.e., such a lift $F$ exists, then $\Pi_{1}$ is weakly equivalent to the diagram $\phi_{F}$ and holim $\left(\mathrm{B} \Pi_{1}\right)$ is weakly equivalent as a space to holim $\left(\mathrm{B} \phi_{F}\right)$. In particular $\pi_{0}$ holim $\left(\mathrm{B}_{1}\right)$ is isomorphic to $\lim ^{1} \phi_{F}[1$, p. 309] and so corresponds bijectively (2.4) to the set of isomorphism classes of $\mathrm{H}^{*}$-clones of $F$. There is a natural map $r_{\mathbf{D}^{\text {op }}} \bar{X} \rightarrow \mathrm{B}_{1}$ and, given a component of holim $\left(\mathrm{B}_{1}\right)$ corresponding to an $\mathrm{H}^{*}$ clone $G$, the problem of lifting this component to a component of $\operatorname{holim}\left(r_{\mathbf{D}^{\mathrm{op}}} \bar{X}\right)^{f}$ is equivalent to the problem of realizing $G$ by a diagram of spaces.

In this way computing $\pi_{0} \operatorname{holim}\left(r_{\mathbf{D}^{\mathrm{op}}} \bar{X}\right)^{f}$ can be broken down into two steps: first, compute the set of lifts of $\bar{X}$ to Ho (and observe that if any such lift exists, the others are exactly its $\mathrm{H}^{*}$-clones and so are enumerated by 2.4); second, determine which of these lifts of $\bar{X}$ to Ho lift further to Sp, and analyze in each case how many of these second-level lifts there are (this can be handled by [7]).

We end with a conjecture related to 1.1.

6.8 Conjecture. Let $\mathcal{X}$ be a p-compact group (perhaps connected) and $\bar{\alpha}_{\mathcal{X}}^{H}$ the diagram in $\mathbf{C o H o}$ which underlies $\alpha_{\mathcal{X}}$. Then the realization space $r \bar{\alpha}_{\mathcal{X}}^{H}$ is connected.

Conjecture 6.8 asserts that the trivial clone of $\bar{\alpha}_{\mathcal{X}}$ is the only one which can be realized as a diagram of spaces, and adds that up to weak equivalence there is only one such realization. Technically, Conjecture 6.8 does not include 1.1; it would be possible for 6.8 to hold and 1.1 to fail if there existed nontrivial $\mathrm{H}^{*}$-clones of $\bar{\alpha}_{\mathcal{X}}$ which could not be realized as diagrams of spaces. In fact we conjecture that this does not happen, and that more generally the $E^{2}$-term of the homotopy spectral sequence for $\pi_{*} \operatorname{holim}\left(r_{\mathbf{D}^{\text {op }}} \bar{\alpha}_{\mathcal{X}}^{H}\right)^{f}$ (see [7, §3] and [1, XI, 6.2]) is trivial away from the $y$-axis.

Remark. Conjecture 6.8 can be proved for the special case in which $\mathcal{X}$ is the 2completion of $G_{2}$ by combining the result of $\S 5$ with an argument along the lines of the one in $[10, \S 7]$. 


\section{REFERENCES}

[1] A.K. Bousfield and D.M. Kan, Homotopy Limits, Completions and Localizations, Lecture Notes in Mathematics vol. 304, Springer-Verlag, Berlin, 1972. MR 51:1825

[2] W. G. Dwyer, The centralizer decomposition of BG, Proceedings 1994 Barcelona Conference on Algebraic Topology (to appear).

[3] - A classification theorem for diagrams of simplicial sets, Topology 23 (1984), 139155. MR 86c:55010a

[4] W. G. Dwyer and D. M. Kan, Realizing diagrams in the homotopy category by means of diagrams of simplicial sets, Proc. Amer. Math. Soc. 91 (1984), 456-460. MR 86c:55010b

[5] W. G. Dwyer and D. M. Kan, Equivariant homotopy classification, J. Pure and Applied Algebra 35 (1985), 269-285. MR 86h:55008

[6] W. G. Dwyer, D. M. Kan, and J. H. Smith, Homotopy commutative diagrams and their realizations, J. Pure and Applied Algebra 57 (1989), 5-24. MR 90d:18007

[7] W. G. Dwyer and D. M. Kan, Centric maps and realization of diagrams in the homotopy category, Proc. Amer. Math. Soc. 114 (1992), 575-584. MR 92e:55011

[8] W. G. Dwyer, H. R. Miller and C. W. Wilkerson, Homotopical uniqueness of classifying spaces, Topology 31 (1992), 29-45. MR 92m:55013

[9] W. G. Dwyer and C. W. Wilkerson, A cohomology decomposition theorem, Topology 31 (1992), 433-443. MR 93h:55008

[10] W. G. Dwyer and C. W. Wilkerson, A new finite loop space at the prime two, J. Amer. Math. Soc. 6 (1993), 37-63. MR 93d:55011

[11] W. G. Dwyer and C. W. Wilkerson, Homotopy fixed point methods for Lie groups and finite loop spaces, Annals of Math. 139 (1994), 395-442. MR 95e:55019

[12] W. G. Dwyer and C. W. Wilkerson, The center of a p-compact group, Contemporary Mathematics 181, Proceedings of the 1993 Čech Conference (Northeastern Univ.), Amer. Math. Soc., Providence, RI, 1995, pp. 119-157. CMP 95:09

[13] S. Jackowski and J. E. McClure, Homotopy decomposition of classifying spaces via elementary abelian p-groups, Topology 31 (1992), 113-132. MR 92k:55026

[14] S. Jackowski, J. McClure and R. Oliver, Maps between classifying spaces revisited.

[15] J. Lannes, Sur les espaces fonctionnels dont la source est le classifiant d'un p-groupe abélien élémentaire, Publications Mathématiques de l'Institut des Hautes Études Scientifiques 75 (1992), 135-244. MR 93j:55019

[16] S. Mac Lane, Categories for the Working Mathematician, Graduate Texts in Mathematics vol. 5, Springer, Berlin, 1971. MR 50:7275

[17] D. Notbohm, Homotopy uniqueness of classifying spaces of compact connected Lie groups at primes dividing the order of the Weyl group, Habilitationsschrift, Göttingen, 1992.

[18] R. Oliver, Higher limits via Steinberg representations, Comm. Algebra 22 (1994), 1381-1393. MR 95b:18007

[19] K. Premadasa, Thesis (Purdue University) 1994.

Department of Mathematics, University of Notre Dame, Notre Dame, Indiana 46556

E-mail address: dwyer.1@nd.edu

Department of Mathematics, Purdue University, West Lafayette, Indiana 47907

E-mail address: wilker@math.purdue.edu 